Mon. Not. R. Astron. Soc. 000, 1-?? (2006) Printed 5 January $2018 \quad$ (MN $2 \mathrm{IT}_{\mathrm{E}} \mathrm{X}$ style file v2.2)

\title{
Invariant manifolds, phase correlations of chaotic orbits and the spiral structure of galaxies
}

\author{
N. Voglis ${ }^{1}$, P. Tsoutsis ${ }^{1,2}$, and C. Efthymiopoulos ${ }^{1}$ \\ ${ }^{1}$ Research Center for Astronomy and Applied Mathematics, Academy of Athens, Soranou Efessiou 4, GR-115 27 Athens, Greece \\ ${ }^{2}$ Section of Astronomy, Astrophysics and Mechanics, Department of Physics, University of Athens, \\ Panepistimiopolis, GR-15784 Zografos, Athens, Greece \\ e-mail:nvogl@academyofathens.gr,cefthim@academyofathens.gr,ptsoutsi@phys.uoa.gr
}

Released 2006 Xxxxx XX

\begin{abstract}
In the presence of a strong $m=2$ component in a rotating galaxy, the phase space structure near corotation is shaped to a large extent by the invariant manifolds of the short period family of unstable periodic orbits terminating at $\mathrm{L}_{1}$ or $\mathrm{L}_{2}$. The main effect of these manifolds is to create robust phase correlations among a number of chaotic orbits large enough to support a spiral density wave outside corotation. The phenomenon is described theoretically by soliton-like solutions of a Sine-Gordon equation. Numerical examples are given in an N-Body simulation of a barred spiral galaxy. In these examples, we demonstrate how the projection of unstable manifolds in configuration space reproduces essentially the entire observed bar-spiral pattern.
\end{abstract}

Key words: galaxies: spiral, structure, kinematics and dynamics

\section{INTRODUCTION}

Any gravitational theory of galactic spiral structure based on orbits (see Contopoulos 2004, pp.497-503, for a review) must provide a model of the way the phases of the orbits, i.e, the angular positions of the apsides, become correlated, so as to reproduce self-consistently the observed spiral pattern. Models for normal spirals based on stable periodic orbits of the $x_{1}$ family, with apocenters aligned along the spiral, were proposed by Contopoulos and Grosbøl (1986, 1988). In such models, the termination of the main spiral is placed near the 4/1 resonance (Contopoulos 1985, Patsis et al. 1991, 1994, Patsis and Kaufmann 1999), while weak extensions may also be found beyond the $4 / 1$ resonance, reaching the corotation region. On the other hand, such models are probably not applicable at all if the spirals are supported mostly by chaotic orbits, as, for example, in the case when a bar is present (Kaufmann and Contopoulos 1996). The dominance of chaotic orbits near and beyond corotation renders these orbits 'instrumental' (Kaufmann and Contopoulos 1996) in successful self-consistent models of barred-spiral galaxies. In particular, chaotic orbits with Jacobi constants exceeding the value at $L_{1,2}$ can circulate in and out of corotation, thus supporting both the spiral and the bar (e.g. the 'hot' population of stars in the N-Body models of Sparke and Sellwood 1987).

A central question in the study of disk galaxies is whether the bar drives spiral dynamics (e.g. Goldreich and Tremaine 1978, Athanassoula 1980, Schwarz 1984, 1985), or the spiral structure is a recurrent collective instability char- acterized by its own independent dynamics (coupled perhaps to the bar dynamics, e.g. Sellwood 2000 and references therein). In order to understand this problem, a relevant key question is what mechanisms generate the spiral pattern and whether such mechanisms work preferably with regular or with chaotic orbits. In the present paper we address the question of mechanisms generating phase correlations of chaotic orbits able to support a spiral pattern.

The set of chaotic orbits near corotation fill stochastically a connected chaotic domain of the phase space. The stochastic character of the orbits could be naively perceived as opposed to any persistent phase correlation among these orbits. However, it is well known in the theory of dynamical systems that the effective randomness exhibited by chaotic orbits is to a certain extent only apparent. This is because of at least two reasons:

i) The loss of information along a chaotic orbit becomes important only for times longer than the Lyapunov time of the orbit (the inverse of the Lyapunov Characteristic Number).

ii) Even for times longer than the Lyapunov times the loss of information in a chaotic domain, due to the exponential stretching of a phase space volume element, which is maximum along one particular local direction, is accompanied by a contraction of the element across the same direction, i.e a gain of information across this direction. Such local directions are determined by the invariant manifolds of unstable periodic orbits in the chaotic domain. The concept of invariant manifolds is a key concept in understanding the 
chaotic dynamics in the corotation region as will be analyzed in section 2 below.

In particular, we shall provide evidence that the unstable invariant manifolds of the short-period unstable periodic orbits, which form two families, one terminating at $L_{1}$ and the other at $L_{2}$ (see section 2), provide a mechanism yielding phase correlations of chaotic orbits that support, precisely, a spiral density wave beyond the bar.We find that the intersections of invariant manifolds with configuration space define patterns quite similar to the spiral pattern of the observed density field. By using an analysis parallel to the analysis given in Voglis (2003), it is theoretically demonstrated (subsection 2.2) that the angular positions of the apocenters of chaotic orbits with initial conditions along an unstable manifold can be given, as a function of time, by a soliton-type solution of a Sine-Gordon equation. This solution is quantitatively accurate only for very small amplitudes of the perturbation. However, even for large amplitudes of the perturbation we find the same qualitative behavior, that is the successive apocenters of chaotic orbits remain correlated for quite long times, despite the positive Lyapunov exponents of these orbits. This phase correlation causes the manifold to support the spiral pattern. We furthermore provide a numerical calculation of invariant manifolds in an N-Body simulation of a barred spiral galaxy. In this calculation, we superpose the figures of the manifolds on the locus of local maxima of the spiral density given directly by the N-body particle distribution projected on the plane of the disc. These two figures show a satisfactory agreement.

A general property of invariant manifolds is that the unstable manifold of one periodic orbit cannot intersect itself or the unstable manifolds of other periodic orbits. When the phase space is compact (i.e. no escapes are possible), the latter property implies also that the manifolds of all the orbits fill densely the phase space and that they are locally parallel to each other (the same property holds also for stable manifolds). We find, with numerical examples, that this geometric arrangement of the manifolds is retained to a large extent even when the phase space is not compact, i.e., some escapes are possible, provided that the rate of escapes is slow. Given this property, we anticipate that the effects shown below for a particular class of manifolds corresponding to simple periodic orbits forming families terminating at $L_{1}$ (or $L_{2}$ ) should in principle be present also in the manifolds of many other families of unstable periodic orbits. Finally, another important property of invariant manifolds is their structural stability, i.e., the manifolds retain their phase-space geometric structure under small perturbations of a system.

The paper is organized as follows: In section 2 we define and calculate the invariant manifolds of unstable periodic orbits in a simple Hamiltonian model describing the dynamics in the corotation region. In particular, we discuss the role played by invariant manifolds in the dynamics of chaotic orbits. Section 3 presents numerical results from an N-Body simulation of a barred spiral galaxy. The manifolds are calculated first in a Poincaré section and then their intersections with the $2 \mathrm{D}$ configuration space of the disc are plotted. These plots are compared to the spiral pattern of the system as derived by the particle distribution on the disc. Section 4 summarizes the main conclusions from the present study.

\section{THEORY}

\subsection{The phase space structure near corotation and the form of invariant manifolds}

The general form of the Hamiltonian of motion in the rotating frame of a disc galaxy with a non-axisymmetric potential perturbation is:

$H\left(r, \theta, p_{r}, p_{\theta}\right) \equiv \frac{1}{2}\left(p_{r}^{2}+\frac{p_{\theta}^{2}}{r^{2}}\right)-\Omega_{p} p_{\theta}+V_{0}(r)+V_{1}(r, \theta)$

where $\left(r, \theta, p_{r}, p_{\theta}\right)$ are polar coordinates and their respective conjugate canonical momenta, and $\Omega_{p}$ is the angular velocity of the pattern. Assuming a bar-like perturbation $V_{1}$, a good first approximation to the Hamiltonian (11) in the neighborhood of corotation is given by (Contopoulos 1978):

$H=\kappa_{*} I_{1}+\alpha_{*} I_{1}^{2}+2 b_{*} I_{1} I_{2}+c_{*} I_{2}^{2}+A_{*} \cos 2 \theta_{2}$

In this formula, $I_{1}$ is the action corresponding to epicyclic oscillations, $\kappa_{*}$ being the respective epicyclic frequency. $I_{2}$ is an $O\left(A_{*}\right)$ correction to the quantity $p_{\theta}-J_{*}$, where $p_{\theta}$ is the angular momentum of an orbit in the inertial frame and $J_{*}$ is the value of $p_{\theta}$ for a circular orbit at the corotation radius of the unperturbed $\left(V_{1}=0\right)$ potential. The angle $\theta_{2}$ is also an $O\left(A_{*}\right)$ correction to the azimuthal angle $\theta$. For simplicity, in the analysis below we set $I_{2} \approx p_{\theta}-J_{*}$ and $\theta_{2} \approx \theta$.

Since the epicyclic angle $\theta_{1}$, conjugate to $I_{1}$, is ignorable in the Hamiltonian (2), this Hamiltonian model is integrable, $I_{1}$ being a second integral independent of and in involution with $H$. However, if any other term $\exp \left(i\left(n \theta_{1}+m \theta_{2}\right)\right)$ of the harmonic expansion of $V_{1}$ is added to the Hamiltonian (2), the Hamiltonian becomes, in general, non-integrable and chaos is introduced to the model. As an example, we may consider the Fourier modes $n=1, m= \pm 2$, which are important near Lindblad resonances. A simple example of a Hamiltonian model including such terms is

$$
\begin{aligned}
H & =\kappa_{*} I_{1}+\alpha_{*} I_{1}^{2}+2 b_{*} I_{1} I_{2}+c_{*} I_{2}^{2}+A_{*} \cos 2 \theta_{2} \\
& -\epsilon\left(\frac{2\left(I_{1}+I_{10}\right)}{\kappa_{*}}\right)^{1 / 2} I_{2}\left[\cos \left(\theta_{1}+2 \theta_{2}\right)-\cos \left(\theta_{1}-2 \theta_{2}\right)\right]
\end{aligned}
$$

The dependence of the Fourier terms $\cos \left(\theta_{1} \pm 2 \theta_{2}\right)$ on the actions $I_{1}, I_{2}$ introduced in Eq.(3) is a simplified model used to demonstrate a number of phenomena relevant to the discussion below, while, in reality, the dependence of any Fourier term of $V_{1}$ on the actions would be determined by the particular functional form of $V_{1} . I_{10}$ is just a smoothing constant to avoid the singularity of the derivative with respect to $I_{1}$. At any rate, the crucial parameter in all these phenomena is the overall size of the perturbation terms $\cos \left(\theta_{1} \pm 2 \theta_{2}\right)$, determined in Eq. (3) by $\epsilon$.

For Jacobi constant values larger than the value at the unstable Lagrangian equilibrium points $L_{1,2}$, there is a family of unstable short-period orbits around $L_{1}$, or $L_{2}$ (Fig. 1, schematic). These orbits form loops of size that increases as the value of the Jacobi constant increases. We denote with $P_{L 1,2}$ the point where a short-period orbit passes from its apocenter. We shall also refer to the orbits themselves as the short period orbits $P_{L 1}$ (or $P_{L 2}$ ). The curves with arrows correspond to the equipotential passing from $L_{1,2}$ and the arrows indicate the sense of flow of orbits inside corotation (prograde, counterclockwise) or outside corotation (retrograde, clockwise). A similar family of stable short-period 
orbits with apocenters $P_{L 4,5}$ exists also for the stable Lagrangian points $L_{4,5}$ for values of the Jacobi constant exceeding the value at $L_{4,5}$.

Now, a visualization of the phase space of the system (3) can be obtained by means of a suitable Poincaré surface of section. Figure $2 \mathrm{a}$ shows the surface of section $\left(I_{2}, \theta_{2}\right)$ corresponding to the successive crossings of the angle $\theta_{1}$ along an orbit with the values $2 n \pi, n=1,2, \ldots$, i.e., at the apocenters of orbits, when $\epsilon$ is very small $\left(\epsilon=10^{-3}\right)$. The basic phase portrait (Fig. 2a) resembles a pendulum phase portrait, with a separatrix dividing the phase space into libration and rotation regions. The fixed points $P_{L 1,2}$ and $P_{L 4,5}$ correspond to the same points as in Fig. 1 . The points $P_{L 1,2}$ exist for a range of values of the Jacobi constant $E_{J}>E_{J, L 1,2}$, while the points $P_{L 4,5}$ exist for a range of values of $E_{J}$, such that $E_{J}>E_{J, L 4,5}>E_{J, L 1,2}$. In the latter case, the phase portrait is divided in two libration domains, around the stable points $P_{L 4,5}$, and two rotation domains, above and below the unstable fixed points $P_{L 1,2}$, in which the orbits make rotations around the galaxy in either the retrograde sense (above $P_{L 1}$, outside corotation), or prograde sense (below $P_{L 1}$, inside corotation). On the other hand, if $E_{J, L 4,5}>E_{J}>E_{J, L 1,2}$, there is an energetically forbidden domain that replaces some of the KAM curves around $P_{L 4}$ and $P_{L 5}$ of Fig. 2a.

Figure $2 \mathrm{a}$ gives initially the impression of being composed only by invariant curves, i.e., by regular orbits. However, a closer look reveals that there is a small degree of chaos in the neighborhood of the unstable points $P_{L 1,2}$. This chaos is clearly manifested by computing the form of the invariant manifolds emanating from $P_{L 1}$ or $P_{L 2}$ (Fig. 2b).

By definition, we call unstable manifold $\mathcal{W}^{U}$ of the short period unstable periodic orbit $P_{L 1}$ the set of points in phasespace with pre-images approaching asymptotically the orbit $P_{L 1}$ as $t \rightarrow-\infty$. Similarly, the stable manifold $\mathcal{W}^{S}$ of $P_{L 1}$ is the set of points in phase-space with images approaching asymptotically the orbit $P_{L 1}$ as $t \rightarrow \infty$. Both sets, $\mathcal{W}^{U}$ and $\mathcal{W}^{S}$ are two-dimensional manifolds embedded in the three-dimensional Jacobi constant hypersurface of the fourdimensional phase-space.

If, now, we work on a particular Poincaré surface of section, such as, for example, the section of the apocenters as in fig.2, the unstable periodic orbit $P_{L 1}$ appears as an unstable fixed point $P_{L 1}$. Furthermore, the two-dimensional manifolds $\mathcal{W}^{U}$ or $\mathcal{W}^{S}$ intersect this section along one-dimensional submanifolds on the two-dimensional surface of section. These submanifolds are the set of points approaching asymptotically the fixed point $P_{L 1}$ as $t \rightarrow-\infty$ or $t \rightarrow \infty$, respectively. In order to distinguish between two-dimensional manifolds in phase space and one-dimensional manifolds in the surface of section, we call the latter apocentric or pericentric manifolds, depending on whether we take the surface of section at the apocenters or pericenters of the orbits respectively. In fact, one can define a surface of section in many different ways, but the definitions adopted here, in terms of the apocenters or the pericenters, are more convenient for the present study.

A well known theorem of dynamics (Grobmann 1959, Hartman 1960) proves that:

a) in autonomous Hamiltonian systems the manifolds on the Poincaré surface of section are invariant, i.e., coincide with their images under the Poincaré map, and b) they approach the fixed point $P_{L 1}$ in the directions tangent to the eigenvectors of the linearized map around $P_{L 1}$. In particular, the unstable manifold is tangent to the eigenvectors associated with the absolutely larger real eigenvalue (say $\lambda_{1}$ ) of the monodromy matrix at $P_{L 1}$, while the stable manifold is tangent to the eigenvectors associated with the absolutely smaller eigenvalue, $\lambda_{2}$, of the same matrix. By the symplecticity property of Poincaré maps we furthermore have $\lambda_{1} \lambda_{2}=1$, thus $\left|\lambda_{1}\right|>1$ and $\left|\lambda_{2}\right|<1$.

Property (b) can be exploited to calculate numerically the invariant manifolds emanating from a particular unstable periodic orbit. Namely, let the functions

$\theta_{2}^{\prime}=F\left(\theta_{2}, I_{2}\right), \quad I_{2}^{\prime}=G\left(\theta_{2}, I_{2}\right)$

represent the Poincaré map of the system under study (the functions $F, G$, are determined numerically). We then calculate, by a Newton-Raphson scheme, the position of one unstable periodic orbit, say $P_{L 1}$, which corresponds to one root $\left(\theta_{2,0}, I_{2,0}\right)$ of the set of equations $\theta_{2,0}=F\left(\theta_{2,0}, I_{2,0}\right)$, $I_{2,0}=G\left(\theta_{2,0}, I_{2,0}\right)$. The monodromy matrix at the fixed point $P_{L 1}$ is defined as

$A=\left(\begin{array}{ll}\frac{\partial F}{\partial \theta_{2}} & \frac{\partial F}{\partial I_{2}} \\ \frac{\partial G}{\partial \theta_{2}} & \frac{\partial G}{\partial I_{2}}\end{array}\right)_{\left(\theta_{2,0}, I_{2,0}\right)}$

where partial derivatives can also be determined numerically. The eigenvalues $\lambda_{1}$ and $\lambda_{2}$, of $A$, are real and reciprocal. We then take a small initial segment $d S$ with one of its endpoints coinciding with the fixed point $P_{L 1}$ and the other endpoint oriented on the direction of the eigenvector associated with one of the eigenvalues $\lambda_{1}$, or $\lambda_{2}$. Finally, we consider a large number of initial conditions of orbits along the segment $d S$. The successive Poincaré map images of the points along the segment associated with $\lambda_{1}$ numerically determine the unstable one-dimensional invariant manifold of $P_{L 1}$. Similarly, pre-images of points along the segment associated with $\lambda_{2}$ determine the stable one-dimensional invariant manifold of $P_{L 1}$.

Figure $2 \mathrm{~b}$ shows a focus near $P_{L 1,2}$ of the apocentric invariant manifolds emanating from $P_{L 1,2}$ for the same model and parameter values as in Fig. 2a. The form of the manifolds of $P_{L 2}$, when they approach the neighborhood of $P_{L 1}$, is characteristic of what is known as 'homoclinic chaos' in the theory of Hamiltonian dynamical systems. Namely, the stable and unstable manifolds have transverse intersections, called homoclinic points. The part of a manifold between any two successive homoclinic points determines a lobe. The successive Poincaré images of the lobes are more and more elongated along the two eigendirections, creating a very complicated homoclinic tangle. That the complexity of the dynamics induced by the homoclinic tangle is the source of chaos in Hamiltonian systems was already pointed out by Poincaré (1892). However, a non-schematic numerical calculation of invariant manifolds in a simple Hamiltonian model was given relatively recently (Contopoulos and Polymilis 1993). It has been established theoretically that this dynamics is isomorphic to the dynamics of the so-called $\Lambda$ - set (e.g. Contopoulos 2004, p. 152), which obeys the Bernoulli shift map. The latter is considered as a paradigm of chaotic system.

The geometric structure of invariant manifolds in phase space is preserved even for large values of the nonlinearity parameter. This effect is shown in Figs. 2c,d. The parameter $\epsilon$ has now a value $10^{-2}$, i.e., ten times larger than in Figs. 
2a,b. As a result, the phase space near $P_{L 1}$ is now largely chaotic, and most rotational invariant curves are destroyed (Fig. 2c). However, the apocentric invariant manifolds emanating from $P_{L 1,2}$ (Fig. 2d) preserve a geometric structure similar to that of Fig. $2 \mathrm{~b}$, except for the fact that the overall vertical scale of Fig. $2 d$ is larger by a factor five from that of Fig. 2b. This implies that, as $\epsilon$ increases, the size of the lobes of the invariant manifolds increases. The lobes extend to bigger distances away from $P_{L 1,2}$.

Now, the unstable invariant manifolds of an unstable periodic orbit, starting at the directions (U,UU), establish a preferential direction of chaotic stretching in phase space in the forward sense of time. In particular, all the orbits with initial conditions inside the area defined by the lobes of one unstable manifold are forced to follow the same preferential direction as that of the manifold. Furthermore, the unstable manifolds of other unstable periodic orbits in the same chaotic domain cannot intersect the unstable manifold of the first orbit, thus they also follow the same directions. Such directions are defined in the neighborhood of every point in the chaotic domain and they serve as the basis of construction of numerical indicators distinguishing between ordered and chaotic orbits (Voglis et al. 1998, 1999, 2002).

Returning to the discussion of manifolds emanating from $P_{L 1}$, the relevant remark regarding the chaotic behavior of orbits near corotation is that, as the lobes of the unstable manifold U, or UU, approach close to $P_{L 2}$, they become more and more elongated, so that they repeatedly cross the straight lines corresponding to the directions S, or SS (Figs. $2 \mathrm{~b}, \mathrm{~d})$. At every such crossing, a lobe goes from the chaotic domain above $P_{L 2}$ (outside corotation), to the domain below $P_{L 2}$ (inside corotation), or vice versa. As a result, all chaotic orbits with initial conditions on, or inside, a lobe repeatedly go inside and outside of the corotation region.

The sequence of these events has a Bernoulli shift dynamics, i.e., it is a very chaotic phenomenon. This fact notwithstanding, we show in the next subsection that the same dynamics imposed by unstable invariant manifolds implies that the phases of chaotic orbits near an unstable manifold are organized along the dominant directions of stretching, due to the area preservation, and they become well correlated. This effect, precisely, serves as the basis of support of a spiral density wave composed by chaotic orbits.

\subsection{Soliton-like motions and phase correlations of chaotic orbits}

Voglis (2003) has shown that in the neighborhood of any resonance in a disc galaxy, the phases of stars with initial conditions close to the separatrix associated with the resonance follow a soliton-type motion. A particular numerical application was given in the case of the Inner Lindblad Resonance, but exactly the same formalism can be applied to other resonances. In this section we shall assume the Hamiltonian to be of the form (3) and describe soliton-type correlations applicable to the orbits near $P_{L 1,2}$.

To this end, we introduce the canonical transformation $\left(\theta_{1}, \theta_{2}, I_{1}, I_{2}\right) \rightarrow\left(\theta_{1}^{\prime}, \theta_{2}^{\prime}, I_{1}^{\prime}, I_{2}^{\prime}\right)$ defined by

$I_{i}=\frac{\partial F}{\partial \theta_{i}}, \quad \theta_{i}^{\prime}=\frac{\partial F}{\partial I_{i}^{\prime}}, \quad i=1,2$

where the generating function $F\left(\theta_{i}, I_{i}^{\prime}\right)$ is chosen in such a way as to eliminate the dependence of the Hamiltonian on the angle $\theta_{1}$ up to terms of order $O(\epsilon)$. Precisely, we have

$$
\begin{aligned}
F= & \theta_{1} I_{1}^{\prime}+\theta_{2} I_{2}^{\prime}+\epsilon\left(\frac{2\left(I_{1}^{\prime}+I_{10}\right)}{\kappa_{*}}\right) \frac{I_{2}^{\prime}}{\kappa_{*}} \times \\
& {\left[\sin \left(\theta_{1}+2 \theta_{2}\right)-\sin \left(\theta_{1}-2 \theta_{2}\right)\right] }
\end{aligned}
$$

Substituting the transformation (6) into the Hamiltonian (3), the Hamiltonian takes the form:

$H=\kappa_{*} I_{1}^{\prime}+\alpha_{*} I_{1}^{\prime 2}+2 b_{*} I_{1}^{\prime} I_{2}^{\prime}+c_{*} I_{2}^{\prime 2}+A_{*} \cos 2 \theta_{2}^{\prime}+O(A \epsilon)(8)$

where primed variables differ by $O(\epsilon)$ terms from their respective non-primed variables, and both quantities $A$ and $\epsilon$ are assumed small. Ignoring terms of order $O(A \epsilon)$, Hamilton's equations of motion in the Hamiltonian (8) can be cast in the form:

$\ddot{\psi}-\omega_{0}^{2} \sin \psi=0$

where $\psi=2\left(\theta_{2}^{\prime}-\pi / 2\right)$ and $\omega_{0}^{2}=-8 c_{*} A_{*}$ (the constant $c_{*}$ is negative, while $A_{*}$ is positive). The first integral of (9) reads

$\frac{\dot{\psi}^{2}}{2}+\omega_{0}^{2} \cos \psi=C$

The quantity (10) represents an approximate integral of motion which, in a strict sense, is valid only for orbits lying on invariant curves such as those of Fig. 2a. In particular, the value of the constant $C=\omega_{0}^{2}$ corresponds to a theoretical separatrix curve passing from $P_{L 1,2}$. Along this curve, the solution of (9) reads

$\tan \left(\frac{\psi}{4}\right)=\exp \left[ \pm\left(\omega_{0} t-k_{0} \xi\right)\right]$

where the parameter $\xi$ is related to the initial phase $\psi_{0}$ of an orbit on the separatrix via $\xi=-\frac{1}{k_{0}} \ln \tan \left(\psi_{0} / 4\right)$, and $k_{0}=\omega_{0} / \omega_{1}$, with $\omega_{1}=\kappa_{*}+O\left(A_{*}\right)$. This choice of $\omega_{1}$ allows us to follow the evolution of the phases $\psi$ for the successive Poincaré consequents of the orbits when $\theta_{1}=\omega_{1} t=2 \pi n$, with $n=1,2, \ldots$, i.e., at the apocenters of the orbits.

Now, the solution (11) can be viewed as a kink, or antikink, soliton-type solution of the Sine-Gordon equation:

$\frac{\partial^{2} \psi}{\partial t^{2}}+\omega_{1}^{2} \frac{\partial^{2} \psi}{\partial \xi^{2}}-2 \omega_{0}^{2} \sin \psi=0$

In Eq.(12), $\xi$ was attributed the status of an independent variable of the problem expressing the initial value of $\theta_{2}$ on the Poincaré surface of section. The reason for this is that the number of stars in a galaxy is large enough so as to expect that the separatrix defined by the apocenters of stars is populated by stars all along its length. We may thus view Eq. (12) as describing the evolution of the phases $\psi$ of a chain of stars populating the separatrix. This is given as a function of the time, $t$, but also of the initial phase of each star, given by $\xi$.

The above analysis was based on a canonical transformation of first order in the perturation $\epsilon$, which also approximates parts of the manifolds as separatrices. The question then arises whether the soliton-type flow of phases induced by Eq. (12) is a good approximation of the true flow of phases along the invariant manifolds as calculated numerically, i.e., without any approximation. In order to explore this question, we first avoid the multiplicity of roots of Eq. (11) with respect to $\psi$ by differentiating this equation with respect to time, that is $\dot{\psi}= \pm 2 \omega_{0} \operatorname{sech}\left(\omega_{0} t-k_{0} \xi\right)$, or 
$\dot{\theta_{2}^{\prime}}= \pm \omega_{0} \operatorname{sech}\left(\omega_{0} t-k_{0} \xi\right)$

In order to compare numerical results with analytical predictions, we then plot the theoretical relation (13) against the numerical evaluation of $\dot{\theta}_{2}$ (by Hamilton's equations from the Hamiltonian (3)) for an ensemble of points being initially in a small segment along the unstable eigenvector pointing in the UU direction, from $P_{L 1}$, and the U direction, from $P_{L 2}$. As shown in Fig. 3, for a relatively large value of the perturbation parameter $\epsilon=10^{-2}$, and at different time snapshots, the numerical solution $\dot{\theta}$ (displayed by dots in Fig. 3), as function of $(\xi, t)$ follows approximately the analytical solution (Eq. (13), displayed by solid line in Fig. 3). In fact, the theoretical solution for $\theta_{2}$ (Eq11) with $\psi=2 \theta_{2}^{\prime}-\pi \simeq 2 \theta_{2}-\pi$ has four non-communicating branches which correspond to the non-communicating parts of a theoretical separatrix that would pass through $P_{L 1,2}$ (Fig. 2a) if chaos was absent, namely a) upper and b) lower part for $0<\theta_{2}<\pi / 2$, and $3 \pi / 2<\theta_{2}<2 \pi$ and $\mathrm{c}$ ) upper and d) lower branch for $\pi / 2<\theta_{2}<3 \pi / 2$. These are merged to two branches for the solution of $\dot{\theta}_{2}$ via Eq.(13). On the contrary, the invariant manifolds allow communication of the branches via the expanding lobes as those shown in Fig. 2 $\mathrm{b}, \mathrm{d}$. This means that when the orbits approach very close to $P_{L 1,2}$ they are deflected to a different branch instead of approaching asymptotically, as $t \rightarrow \infty, P_{L 1}$ or $P_{L 2}$. This means that at, any time $t$, the motions of the manifold points can be followed only within some limits $\xi_{\min } \leqslant \xi \leqslant \xi_{\max }$ with $\xi_{\max }-\xi_{\min }$ large, but not infinite as in the integrable case. Since particles jump to a different branch of the equation (13), than the branch they were initially, their phases $\theta_{2}$ are mixed in time. In order to circumvent this problem, we redefine the value of $\xi$ for each orbit whenever the orbit changes branch, according to Eq.(11), with $\psi=2 \theta_{2}-\pi$ and $\theta_{2}$ equal to its value at the particular moment when the particle changes branch.

The solitary motions shown in Fig. 3 correspond to a correlated motion of the successive apocenters of chaotic orbits with initial conditions along the manifold. This phenomenon, along with the physical meaning of the changes of branches, will be discussed in subsection 2.3 below. Furthermore, we find numerically that this correlation persists to a large extent even for relatively large values of the perturbation $\epsilon$ as a consequence of the structural stability of the invariant manifolds. It is worth examining the consequences of persisting phase correlations for the dynamics induced by chaotic orbits in the corotation region.

\subsection{Mapping of the invariant manifolds in configuration space and the form of spiral arms}

As described in subsection 2.1, the apocentric or pericentric manifolds are one-dimensional objects embedded in a three dimensional space of constant energy. This implies that they have one-dimensional projections in any plane of this space. In the sequel, besides the surface of section $\left(\theta_{2}, I_{2}\right)$, or $\left(\theta, p_{\theta}\right)$, we shall consider the one-dimensional projections of manifolds in the physical, or configuration space represented, e.g., by the canonical position variables $(r, \theta)$, or $x=r \cos \theta$, $y=r \sin \theta$. This allows to compare the topological charac- teristics of the manifolds to morphological characteristics of the simulated galaxy.

In order to understand the geometric connection of the surface of section to the configuration space, a key remark is that, in the corotation region, the surface of section $\left(I_{2}, \theta_{2}\right)$ and the configuration plane $(r, \theta)$, or $x=r \cos \theta, y=r \sin \theta$, are nearly isomorphic. This isomorphy is shown schematically in Fig. 4. The domains denoted A,B,C,D in Fig. 4a are mapped to the domains $\mathrm{A}^{\prime}, \mathrm{B}^{\prime}, \mathrm{C}^{\prime}$, and $\mathrm{D}^{\prime}$, respectively, in Fig. 4b. The mapping is derived as follows: If the origin of epicyclic angles $\theta_{1}$ is selected in such a way so that the Poincaré section points $\left(I_{2}, \theta_{2}\right)$ for $\theta_{1}=2 n \pi, n=1,2, \ldots$ correspond to successive apocentric passages of an orbit, we have $\dot{r}=0$ at every section point. Replacing $p_{\theta} \simeq I_{2}+J_{*}$, $\theta \simeq \theta_{2}$ in the general form of the Hamiltonian (Eq.(1)), the equation of the Jacobi constant $H\left(r, \theta, p_{r}=0, p_{\theta}\right)=E_{J}$ may be solved for the variable $r$, yielding $r$ at the apocenters as a function of $\theta, p_{\theta}$ (or $\left.I_{2}, \theta_{2}\right)$ and of the constant $E_{J}$. One readily finds that for potential functions corresponding to the usual form of the rotation curve expected in a barred galaxy, the apocentric radius $r$ is a monotonically increasing function of $p_{\theta}$ both inside and outside corotation. In the inner parts of the galaxy we expect that the velocity of circular orbits raises as $v_{c} \propto r$ thus $r \propto p_{\theta}^{1 / 2}$. On the other hand, beyond corotation the rotation curve is nearly flat up to a distant radius, i.e., $v_{c} \sim$ const, yielding $r \propto p_{\theta}$. Finally, in the Keplerian limit $v_{c} \propto r^{-1 / 2}$, thus $r \propto p_{\theta}^{2}$. These relations are not seriously distorted by the inclusion of epicyclic oscillations. We shall see in the next section that these monotonic relations are verified by exact calculations in an N-Body system.

Because of the monotonic relation of $r$ and $p_{\theta}$, phase portraits in the space $\left(p_{\theta}, \theta\right)$, or $\left(I_{2}, \theta_{2}\right)$, can be mapped to nearly-isomorphic portraits in the space $(r, \theta)$, where $r$, calculated as indicated above, always represents a local apocentric distance of an orbit.

Figures $4 \mathrm{a}, \mathrm{b}$ show the correspondence between the directions of the stable and unstable manifolds emanating from $P_{L 1,2}$ in the section $\left(I_{2}, \theta_{2}\right)$ (Fig. 4a) and the same directions in configuration space $(x, y)$ (Fig. $4 \mathrm{~b})$. In particular, the unstable manifolds $U$, pointing upwards in Fig. 4a, are mapped to the directions $U$ of Fig. 4b. In the separatrix limit, the unstable manifold $U$ joins smoothly the stable manifold $S$, while the unstable manifold $U U$ joins smoothly the stable manifold SS. Furthermore, the branch US does not communicate with the branch UUSS. We emphasize that such a theoretical separatrix can be constructed for any value of the Jacobi constant at which the periodic orbit $P_{L 1}$ exists, i.e., the separatrix of Fig. $4 \mathrm{~b}$ should not be confused with an equipotential passing through the equilibrium point $L_{1}$. The separatrix gives the slow drift of apocenters along the branch US, or UUSS, that implies a slow drift of the guiding centers of epicyclic motion for all orbits with initial conditions on these two branches. These separatrices do not coincide with the equipotential curves passing through $L_{1}, L_{2}$ even in the limiting case when $P_{L 1}, P_{L 2}$ tend to $L_{1}, L_{2}$. In this respect, the manifolds shown in Fig. $4 \mathrm{~b}$ are in a sense parallel to the 'critical ergos curves' of LyndenBell and Barot (2003), but at a higher value of the Jacobi integral. By an elementary analysis of the linearized flow in the neighborhood of the $P_{L 1,2}$ orbits (named 'Lyapunov orbits'), Romero-Gomez et al. (2006) gave plots similar to 
Fig. 4b. In view of the geometry of the separatrix, these authors identify the manifolds as responsible for creating a ring structure in barred galaxies like NGC1326. They also point out the robustness of these objects, called by them 'flux rings'.

In fact, as shown in the previous subsection, the robustness of the phase flow along a manifold is a consequence of the soliton-type motion of the phases of a chain of orbits populating the manifold. This is shown in Figs. 4c,d, and $4 \mathrm{e}, \mathrm{f}$, which show the invariant manifolds of Fig. $2 \mathrm{~d}$ in phase space and in configuration space after 4 iterations of an initial segment $d S=10^{-4}$, (Figs. 4c,d) and after 12 iterations (Figs. 4e,f). The correspondence in the form of manifold lobes in configuration and phase space is clearly visible in these figures.

We emphasize that each point of figures $4 \mathrm{~d}$,f corresponds to a position of apocenter of a chaotic orbit with initial conditions along the manifolds. These positions change in time, for each particular orbit, following the soliton-type flow described in subsection 2.2. However, the pattern defined by the manifold remains time-invariant. Furthermore, as the lobe oscillations become of larger and larger amplitude, along the successive iterations of the initial $d S$, the manifold moves far away from the separatrix. Given that the sense of rotation of the galaxy is counter-clockwise, we see that the outward branches $U$ of the unstable manifolds emanating from $P_{L 1,2}$ define trailing spiral arms beyond $P_{L 1,2}$. This phenomenon will be studied in detail in the next section. In the same time, the inward directions UU support the bar, i.e., the same chaotic orbits with initial conditions along the manifolds support both the spiral pattern and the bar.

The opening of the manifold lobes is larger and faster when $\epsilon$ increases. We shall see however, in the next section, that the near-isomorphy between phase and configuration portraits is preserved for quite large perturbations. In particular, in order to study how far can extend the manifold spirals, we present a numerical example referring to an $\mathrm{N}$ Body simulation of a barred spiral galaxy. It is then possible to show that the invariant manifolds $U$ generate patterns that closely follow the form of the N-Body spiral pattern of the system.

\section{NUMERICAL SIMULATIONS: COMPARISON OF INVARIANT MANIFOLDS AND SPIRAL ARMS}

Figure 5a shows one characteristic snapshot (for $t=47$ in units of the half-mass crossing time) of an N-Body simulation of a rotating barred - spiral galaxy. A series of such simulations with $N=130000$, is presented in Voglis et al. (2006). These experiments are produced with a smooth potential field code which is an improved version of the code of Allen et al. (1990). The initial conditions for a rotating system are produced from the end-state of the so-called $Q$-model in the simulations of Voglis et al. (2002). This is initially a triaxial configuration produced by a collapse process with cosmological initial conditions consistent with the standard $\Lambda-\mathrm{CDM}$ model. The initial model has an almost zero spin parameter $\lambda$ (Peebles 1969). In order to increase the total angular momentum of the model, while respecting its energy and the scalar virial theorem, the following velocity re-orientation process is implemented: At a given snapshot of N-body evolution of the Q-model $\left(t_{Q}=100\right)$, the velocity component $v_{y z}$ of each particle, where the plane $(y, z)$ is the plane of intermediate - longest axes, is re-oriented so as to become perpendicular to the current cylindrical radius $r_{y z}$ of the same particle. After the re-orientation, the velocity components $v_{y z}$ of all particles point to the direction of rotation clockwise with respect to the $x$ - axis, which thus becomes the rotational axis of the system. This is the way plots are presented in Voglis et al. 2006. In the plots below, however, like Fig. 5a we have reversed the sense of the $\mathrm{x}$-axis so that the pattern in these figures is shown to rotate counterclockwise (this is the most frequent choice in the literature when discussing bar or spiral dynamics).

The so-produced system, called QR1, is let to evolve by the N-Body code until a time $t_{Q R 1}=20$ (in units of the half-mass crossing time), and then the same velocity reorientation process is implemented. The new system, QR2, is left to evolve again by the N-Body code until $t_{Q R 2}=20$, when the velocity re-orientation process is implemented once time. This process can yield different rotating systems which have the same binding energy, and nearly the same virial equilibrium, but they have consecutively higher and higher amounts of angular momentum.

A detailed analysis of the regular and chaotic orbital content as well as of the evolution of the above systems is presented elsewhere (Voglis et al. 2006). We only mention here the results relevant to the analysis below, which refer to the long-time evolution of the QR3 experiment, i.e., the experiment with initial conditions provided by three consecutive applications of the velocity re-orientation algorithm:

a) The initially triaxial configuration has developed a thick disc structure, with a thickness ratio of the order of 0.2. The projection of the system on the disc plane (e.g. Fig. 5a) clearly shows a bar as well as a grand design spiral pattern. In most snapshots the calculated spiral pattern speed has nearly the same value as the bar speed. The spiral amplitude, however, is variable, ranging from a maximum 2 or 3 times smaller than the amplitude of the bar to a minimum that is nevertheless always above the threshold of statistical noise.

b) In some snapshots the spiral looks detached from the bar. Most snapshots, however, manifest a continuity of the pattern between the bar and the spiral.

c) The orbits of all the particles are analyzed as regards their regular, or chaotic character, by a combination of efficient numerical chaos indicators (Voglis et al. 2002). Let us note that the orbital analysis of a particular snapshot is possible because the code provides an expansion of the potential in terms of a set of basis functions, making thereby possible the explicit calculation of orbits, variational equations etc. The main results are: i) The mass in chaotic motion is found to be on the level of $60 \%$ to $65 \%$ of the total mass, and ii) when particles in chaotic or ordered orbits are plotted separately, the spiral arms are found to be composed practically only by chaotic orbits. On the contrary, the bar is composed by both regular and chaotic orbits.

At the snapshot $t=47$, the bar-spiral structure is clearly seen by simple visual inspection (Fig. 5a). All distances in this figure were divided by the half mass radius of the system, which is taken as the unit of length. The 
thick dots show the positions of local maxima of the projected density on the $(y, z)$ plane. This is done by splitting the distribution of points in concentric rings $r, r+\Delta r$, where $\Delta r=0.2$, and calculating the angles of local density maxima within each ring.

Figure 5b shows the distribution of the values of the Jacobi constant for the set of particles which are located within small projected area elements $r \Delta r \Delta \theta$, with $\Delta \theta=2 \pi / 26$ around the values $(r, \theta)$ corresponding to the thick dots of Fig. 5a. The energy units are derived by the numerical code length and time units, i.e., setting the mass of each particle equal to $m_{p}=1$. In these units, the half-mass crossing time is $t_{h m c t}=1.1085 \times 10^{-4}$, the half-mass radius is $R_{h}=9.26 \times 10^{-2}$. The root-mean square velocity is found to be $v_{r m s}=732.6$. The pattern speed (estimated numerically by the rate of angular displacement of the density maxima of the pattern), is equal to $\Omega_{p}=2 \pi / 9.43$ in units of $1 / t_{h m c t}$, i.e., the period of pattern rotation is about ten times larger than the half-mass crossing time (which corresponds to a typical period of the particles' orbits). This means that the rotating pattern can be safely characterized as an evolving density wave.

Another approximation in the calculation of Fig. 5b is the fact that the kinetic energy of a particle includes a $v_{x}^{2} / 2$ term, which corresponds to the kinetic energy of the velocity component perpendicularly to the disc. This small term (a few percent of the total kinetic energy) is substracted from the evaluation of the Jacobi constant, since, for most particles, the motion perpendicularly to the disc is effectively decoupled from the motion on the disc plane. We thus approximate the Jacobi constant for each particle by:

$E_{J}=\frac{1}{2}\left(v_{y}^{2}+v_{z}^{2}\right)-\Omega_{p}\left(z v_{y}-y v_{z}\right)+V(x, y, z)$

where the function $V(x, y, z)$ is calculated by the series of basis functions of the N-Body code.

Figure 5b shows the distribution of Jacobi constants, with the above conventions, for the particles located in the maxima shown in Fig. 5a, where, in addition, we only count the particles in rings beyond the value $r=1$, marking, approximately, the end of the bar. Thus, the distribution of Fig. $5 b$ represents essentially the distribution of values of the Jacobi constant for particles located along the spiral arms. The peak of this distribution is at a value $E_{J}=-1.12 \times 10^{6}$, which is slightly above the value, $E_{J, L 1}=-1.133 \times 10^{6}$.

As described in subsection 2.1, the apocentric manifolds are one-dimensional manifolds in the three-dimensional subspace $\left(r, \theta, p_{\theta}\right)$ of the entire four-dimensional phase space of the system. Figure 6 shows a calculation of the unstable apocentric manifolds emanating from the periodic orbit $P_{L 2}$ in the potential of the system of Fig. 5a (with initial conditions $\left.\theta=3.2247, p_{\theta}=1.194\right)$. Three different projections of these manifolds are shown, namely a) on the surface of section $\left(\theta, p_{\theta}\right), \dot{r}=0, \ddot{r}<0$ (Fig. $\left.\left.6 \mathrm{a}\right), \mathrm{b}\right)$ on the plane $\left(r, p_{\theta}\right)$ (Fig. 6b) and c) on the configuration space $(r, \theta)$ (Fig. 6c). The Jacobi constant has the value $E_{J}=-1.125 \times 10^{6}$, close to the maximum of the distribution of Fig. 5b. (We only examine theoretical orbits with $x=0$, i.e., lying on the disc plane). Fig. 6 shows the seventh Poincaré map image of two initial segments of length $d S=10^{-4}$ on the Poincaré section, tangent to the unstable directions $\mathrm{U}$ and $\mathrm{UU}$ respectively, at the fixed point $P_{L 2}$. As a property of the manifold, the seventh image of the initial $d S$ contains also all the previous images from the first to the seventh. This choice of initial length was made by the criterion that the numerically calculated direction of the segment after its first iteration coincides with the eigendirection as calculated by the monodromy matrix of the periodic orbit $P_{L 2}$ up to 3 significant digits. Furthermore, each segment is populated with 10000 equally spaced points of initial conditions. Figure $6 \mathrm{c}$ shows the calculated projections of the apocentric manifolds $U$ and UU $(2 \times 10000$ points $)$ as well as their symmetric manifolds with respect to the center $y=z=0$, which are the manifolds emanating from $P_{L 1}$.

Figure $6 \mathrm{a}$ is different from the simple picture of manifolds shown in Figs. 2b,d in many aspects. A first difference is that the $\theta$ value of $P_{L 2}$ is close to the value $\theta=\pi$, i.e., shifted by $\pi / 2$ with respect to Figs. $2 \mathrm{~b}, \mathrm{~d}$. This is because at the snapshot $t=47$, the bar is oriented almost horizontally (Fig. 5a). But the most serious difference is that the manifolds of Fig. 6a are broken into disjoint pieces. This breaking of the image of the manifold on the surface of section is an artifact caused by the particular choice of surface of section. This can be exemplified with the help of Figs. 7a,b,c,d which refer to the same manifold, but calculated for six iterations of the initial $d S$, in order to obtain a clearer figure. Figures $7 \mathrm{c}, \mathrm{d}$ show the time evolution of two nearby orbits one of which gives the last point, $\mathrm{P}$, of a disjoint piece of the manifold of Fig. 7a, while the other orbit gives the first point $P^{\prime}$ of the next disjoint piece of the manifold in the same figure. We notice that while the orbits are really nearby (Fig. $7 \mathrm{~d}$ ), the first orbit has a local maximum of $r(t)$ at the point $P$, while the second orbit has no local maximum of $r(t)$ at times near the time of the $P$-maximum. In fact, the second orbit yields a local maximum much later, at the point $P^{\prime}$ (Fig. $7 \mathrm{~d})$, thus breaking the continuity of Poincaré intersections in Fig. 7a between the points $P$ and $P^{\prime}$. The same phenomenon is also shown in Fig. 7c, where we clearly see that, while the first orbit on the surface of section $(\dot{r}=0, \ddot{r}<0)$, represented by the line $\dot{r}=0$ in Fig.7c, intersects the line $\dot{r}=0$ at $\mathrm{P}$, the second orbit passes close to the section line, but without intersecting this line.

We emphasize that the above effect is an artifact of the particular choice of surface of section, which is not a true Poincaré section (Poincaré 1892). In fact, it is hardly possible to find any surface of phase space in the above model that can be proven to be a true Poincaré section. Even so, the invariant manifold surfaces are continuous in phase space, and it is only their intersections with the particular surface of section which are discontinuous. Furthermore, the basic topological property of invariant manifolds, namely the fact that they cannot intersect themselves, is also preserved in the surface of section images of the manifold shown in Figs. $6 \mathrm{a}$ or $7 \mathrm{a}$. This fact implies that, as the number of iterations increases, i.e., when the manifold is calculated for longer times, the manifolds fill the connected chaotic domain embedding the fixed points $P_{L 1,2}$ in the surface of section. This tendency is clearly observed by comparing Fig. 6a, which depicts the manifold after only one more iteration than Fig.7a. In Fig.6a the manifold crosses a larger area in the chaotic domain and it starts showing the effect discussed in section 2 , i.e., it defines preferential directions in the surface of section, given by many manifold segments forming narrow bundles 
in almost parallel directions. Such bundles are clearly visible in both Figs. 6a and 7a.

Let us return to the three projections of the apocentric manifolds on the three planes $\left(\theta, p_{\theta}\right),\left(r, p_{\theta}\right),(r, \theta)$ shown in Figs. 6 a, b, c, respectively. The projection of the apocentric manifolds on the plane $\left(r, p_{\theta}\right)$ shows an impressively narrow correlation between $r$ and $p_{\theta}$. For every branch (U, or UU) there is a monotonic (on average) dependence of $r$ on $p_{\theta}$. This is a numerical verification, in the case of the N-Body experiment, of the monotonic relation between $r$ and $p_{\theta}$, that was discussed in subsection 2.3 .

A direct result of this monotonic (on average) dependence (of the apocentric distances $r$ and the angular momentum $p_{\theta}$ of the orbits on the invariant manifolds) is that causes a near-isomorphy between the projection of apocentric manifolds in phase space $\left(\theta, p_{\theta}\right)$ (shown in Fig. 6a) and in the configuration space $(r, \theta)$ (shown in Fig. 6c).

A main effect caused by this near-isomorphy is that the bundles of preferential directions of the apocentric manifolds that appear as almost straight lines in Fig. 6a, appear as spiral lines in Figs. 6c. In other words the loci of preferential directions defined by the apocentric manifolds define a geometric pattern on the configuration space that, except for some details, has the general form of a grand design spiral.

It should be stressed that all the points of the apocentric manifolds in Figs. 6, 7a,b correspond to local apocentric passages of the respective orbits. Thus, one single orbit does not describe continually in time the manifolds shown in these figures. But if we take a chain of stars that densely populate the manifolds, this chain defines a flow along the manifold that keeps the apocenters of chaotic orbits correlated for quite long times. This correlation was modelled theoretically in subsection (2.2) as a soliton-type flow of the successive positions of the apocenters of chaotic orbits.

In Fig. 8 the positions of maxima of the particle distribution along the N-body spiral arms (Fig. 8a, same as Fig. 5 a) are superposed on the spiral patterns generated by the preferential directions of the unstable invariant manifolds of the $P_{L 1,2}$ family (plotted for three different values of the Jacobi constant in Figs. 8b, c, d, which are in a narrow band of values larger than the Jacobi constant at the Lagrangian points $L_{1}, L_{2}$, and close to the peak of the distribution of the energies of Fig. 5b). It is immediately seen that the manifold spirals are very closely aligned to the real spiral arms. The images of the apocentric manifolds on the configuration space, for the three different values of $E_{J}$, are similar to each other, implying that their superposition enhances the overall spiral pattern induced by the manifolds.

The fact that the projections of apocentric manifolds in the configuration space are well-correlated with the maxima of the spiral density is remarkable, because the form of the manifolds reflects topological properties of the underlying chaotic phase space, while the density maxima are related to the particles' (chaotic) motion in configuration space. We can make a number of comments pointing to the theoretical understanding of this phenomenon:

i) We have compared the distribution of all the particles in chaotic orbits of the system along the Jacobi constant axis given in Voglis et al. 2006 (Fig.15 therein) with the distribution of the particles shown in Fig. 5b. The two distributions are quite similar, and their maxima are projected at about the same value of $E_{J}$, which is very close to the band of values of $E_{J}$ for which the manifolds of Fig. 8 are calculated. This means that the backbone of the spiral arms is related to chaotic orbits with initial conditions close to the apocentric manifolds of figure 8. Particles with values of the Jacobi integrals much different than those near the maximum of the distribution of Fig. 5b partly contribute to the density along the spiral arms. However, we have checked that most of these particles contribute to the density of the axisymmetric background.

ii) Near the bundles of preferential directions, the manifolds act, across these directions, as attractors of the orbits, since any element of an area-preserving phase flow has to contract in the direction across the manifolds in order to compensate for the strong stretching along the manifolds. In fact, the stretching and twist properties of the manifolds are far from being uniform along their length. The attraction across the manifold, as well as the organization of phases, is stronger where the stretching along the manifold is also stronger. This mechanism is more effective along bundles of preferential directions, while it is less effective near the turning points of the manifold. These phenomena are similar to the 'stickiness' phenomena caused by invariant manifolds in model Hamiltonian dynamical systems (e.g. Efthymiopoulos et al. 1997, Contopoulos et al. 1999).

iii) Finally, another property of the manifolds is that they are recurrent (Contopoulos and Polymilis 1993), i.e., the manifold lobes return many times near the points $P_{L_{1,2}}$ even if they temporarily go to large excursions away from $P_{L_{1,2}}$. This mechanism enhances further the density along the segments of strong stretching, and also agrees with the maxima of the density appearing preferably along bundles of the manifold. The recurrence of the manifolds is particularly important in the system under study, because the phasespace is non-compact and the recurrence of chaotic orbits cannot be established by the Poincaré recurrence theorem which is applicable only to compact systems. It is remarkable that the recurrence of the manifolds can create new stickiness effects (recurrent stickiness), leading to further accumulation of points in the sticky region (Contopoulos and Harsoula 2006).

Figure 9 shows a comparison of invariant apocentric manifolds with real spirals at three different time snapshots of the evolution of the N-Body experiment, namely $t=18$ (Figs. 9a,b), $t=47$ (Figs. 9c,d) and $t=74$ (Figs. 9e,f). (The manifolds are plotted after eight iterations, when the spread of numerical points along the manifold is still small enough to allow for a coherent visualization of the manifold).

The main difference in the system at these three snapshots is that, as $t$ increases, the pattern speed $\Omega_{p}$ of the bar has a tendency to decrease as a result of torques exerted to the bar by the rest of the system and the angular momentum transference outwards. The decrease of $\Omega_{p}$ is significant, of order $20 \%$, between $t=18\left(\Omega_{p}=0.8037\right)$ and $t=47\left(\Omega_{p}=0.6662\right)$, while it is less important (about $6 \%$ ) between $t=47$ and $t=74\left(\Omega_{p}=0.6282\right)$.

As the system slows down, the position of $L_{1,2}$ moves outwards and the Jacobi constant $E_{J, L_{1,2}}$ increases. The unstable manifolds in Figs. 9b,d,f are calculated for the periodic orbit of the corresponding $P_{L 1,2}$ family at the Jacobi constant values $E_{J}=-1.25 \times 10^{6}, E_{J}=-1.131 \times 10^{6}$ and $E_{J}=-1.085 \times 10^{6}$, respectively. These values are slightly larger than the Jacobi constant values at the re- 
spective Lagrangian points $L_{1,2}$, namely $E_{J}=-1.26 \times 10^{6}$, $E_{J}=-1.133 \times 10^{6}$ and $E_{J}=-1.088 \times 10^{6}$.

Clearly, as the pattern speed of the bar decreases, the manifolds become more and more open, that is they extend to larger distances beyond corotation under the same number of iterations. Furthermore, Figs. 9b,d,f show that the agreement of the apocentric manifold with the spiral pattern is good precisely in the regions where the manifold segments accumulate in bundles of preferential directions. The overall connection of the manifold's spiral geometries is generally visible in all three figures, except for a weak spiral extension in Fig. 9a corresponding to the outermost points of the spiral maxima of Fig. 9b (this is probably a transient effect).

We conclude that as the manifolds become more and more open, the bundles of preferential directions become broader, but also the real spiral becomes weaker and less clearly defined. A detailed explanation of these phenomena is necessary, since they all point to the direction of a clear connection between the dynamics induced by invariant manifolds and the dynamics of real spiral arms. In particular, given that the locus of apocenters defined by the manifolds is the place where stars spend a large part of their radial period, we conclude that the projections of the apocentric manifolds reveal a narrow dynamical connection between manifolds and spiral arms.

Finally, Fig. 10 shows a calculation of a number 300 of individual stellar orbits (plus another 300 symmetric orbits) with initial conditions along the unstable direction of the manifolds of Fig. $8 \mathrm{~b}$. The twelve different panels correspond to twelve successive positions of the pericenters (white thick dots) and apocenters (black thick dots) of these orbits. The first panel (left-top) is when the apocenters start leaving the neighborhood of $P_{L 1, L 2}$. This figure summarizes the previous conclusions from the point of view of the orbital flow. Namely, we observe that the phases of these orbits remain very well correlated for nine radial periods, and to some extent for another three periods. Furthermore, apocenters are preferably aligned along the spiral pattern, while pericenters are located mostly in a double ring-like locus surrounding the bar and the stable Lagrangian points $L_{4}, L_{5}$. It is remarkable that inside the corotation radius pericenters and apocenters cooperate to support a shell of chaotic orbits surrounding the bar. Outside the corotation radius, for some azimuthal extent from the Lagrangian points $L_{1}$, $L_{2}$, pericenters and apocenters also cooperate to form an enhanced initial arc of the spiral arm. For larger azimuthal angles, however, the pericenters form an arc surrounding the stable Lagrangian points $L_{4}, L_{5}$, while the apocenters form a more open arc following the spiral arms.

Thus, we conclude that the apocentric manifolds are more important than pericentric manifolds, for the formation of spiral arms, not only because of the longer time intervals spent by the orbits near the apocenters than near the pericenters, but also because only the apocentric manifold is responsible for the extension of the spiral arms to large distances from the corotation radius.

\section{CONCLUSIONS}

We have explored the role of the invariant manifolds emanating from simple unstable periodic orbits (forming families terminating at $L_{1}$,or $L_{2}$ ) in producing a mechanism of phase correlations between chaotic orbits beyond corotation in a barred spiral galaxy. In particular, we define the apocentric (or pericentric) one-dimensional manifolds as the intersections of the phase space manifolds by the surface of section of the apocenters (or the pericenters). A theoretical analysis is made, based on a theory of soliton-type motions of phases (Voglis 2003) of orbits with initial conditions along the apocentric manifolds. A numerical analysis is also carried out, referring to the calculation of invariant apocentric manifolds in the potential of an N-Body simulation of a barred spiral galaxy. In this case we explore the connection between the manifolds and the overall dynamical features of the N-Body system. Our main conclusions are as follows:

1) While every orbit with initial conditions on the unstable apocentric manifolds of the $P_{L 1,2}$ unstable periodic orbits is chaotic, the phases (the positions of the apocenters) for a chain of particles with initial conditions populating the manifold remain correlated for quite long times. This correlation can be approximated by a soliton-type motion for the phases. This is true not only for small amplitude of the perturbation, but also, to a large extent, for large amplitude of the perturbation.

2) The portraits of phase space, as described by the apocentric manifolds, have nearly-isomorphic portraits into configuration space. These portraits are described in subsection 2.3 as well as in Fig.6. In particular, for small values of the perturbation, the apocentric manifolds form a double-ring structure surrounding the bar and the stable Lagrangian points $L_{4}, L_{5}$. But when the perturbation is strong enough, the images of the manifolds in configuration space extend to large distances beyond corotation, and they take the form of trailing spiral arms.

3) A comparison of unstable invariant apocentric manifolds with the density maxima of an N-Body experiment along the spiral shows an overall connection of the manifold spirals with the N-Body spirals. In particular, the two sets are closely aligned in all regions where the manifold segments form narrow bundles along preferential directions of strong stretching.

4) As long as the galaxy's pattern slows down, the manifolds become gradually more open and the bundles of preferential directions become broader, but still the connection between manifold and spiral geometry is preserved. On the other hand, the amplitude of the spiral becomes also weaker, and its shape less clearly defined. In conclusion, we find a clear dynamical connection between the loci of apocenters defined by the manifolds of unstable periodic orbits and spiral arms. In this respect, the apocentric manifold is narrowly associated with the positions of maxima of the density along the spiral arms. Furthermore, this connection is preserved for an important fraction of the simulated galaxies' lifetimes.

As a final remark, we may revisit the question posed in the introduction on whether the above mechanism can help clarify the role of the bar in driving the dynamics of spiral arms. Our findings suggest that, through the manifolds, the bar can indeed initiate a spiral structure. Notice that the unstable manifolds are asymmetric in configuration space 
with respect to the bar axis (fig.4) and, as shown in section 2 , they can initiate trailing spirals, provided that the bar is strong and/or rotates fast enough to create a large effective perturbation near corotation. Furthermore, this mechanism exploits the relative flexibility of chaotic orbits to exhibit large excursions in phase space while remaining correlated as regards their phases. On the other hand, a spiral pattern initiated by a bar through the above mechanism amplifies the effective perturbation even more, a fact that acts additively to the whole phenomenon, which is based on chaotic orbits. These points, which may prove to be crucial for the longevity of the induced spiral pattern, are a subject of current research.

Acknowledgements: This project was supported in part by the Empeirikion Foundation. Stimulating discussions, as well as a very careful reading of the manuscript by Prof. G. Contopoulos are gratefully acknowledged.

\section{REFERENCES}

Allen, A.J., Palmer, P.L., and Papaloizou, J, 1990, Mon. Not. R. Astr. Soc. 242, 576.

Athanassoula, E.: 1980, Astron. Astrophys. 88, 184.

Contopoulos, G.: 1978, Astron. Astrophys. 64, 323.

Contopoulos, G.: 1985, Comments on Astrophysics 11, 1.

Contopoulos, G. and Grosbøl, P.: 1986, Astron. Astrophys. 155, 11.

Contopoulos, G. and Grosbøl, P.: 1988, Astron. Astrophys. 197, 83.

Contopoulos, G. and Polymilis, C.: 1993, Phys. Rev. E47, 1546.

Contopoulos, G and Harsoula, M. : 2006 (in preparation). Contopoulos, G., Harsoula, M., Voglis, N., and Dvorak, R.: 1999, J. Phys. A 32, 5213.

Contopoulos, G.: 2004, "Order and Chaos in Dynamical Astronomy", Springer-Verlag.

Goldreich, P. and Tremaine, S.: 1978, Astrophys. J. 222, 850.

Efthimiopoulos, Ch., Contopoulos, G., Voglis, N. and Dvorak, R.: 1997, J. Phys. A: Math. and Gen. 30, 8167.

Grobman, D.M., 1959: Dokl. Akad. Nauk SSSR 128, 880.

Hartman, P., 1960: Proc. Am. Math. Soc. 11, 610.

Kaufmann, D.E. and Contopoulos, G.: 1996, Astron. Astrophys. 309, 381.

Lynden-Bell, D. and Barot, J.M.: 2003, in G. Contopoulos and N. Voglis (Eds) "Galaxies And Chaos", Lect. Notes. Phys. 626, Springer-Verlag, pp30-43.

Patsis, P.A., Contopoulos, G. and Grosbøl, P.: 1991, Astron. Astrophys. 243, 373.

Patsis, P.A., Hiotelis, N., Contopoulos, G. and Grosbøl, P.: 1994, Astron. Astrophys. 286, 46.

Patsis, P.A. and Kaufmann, D.E.: 1999, Astron. Astrophys. 352, 469.

Peebles, P.J.E., 1969, Astrophys. J 155, 393.

Poincaré, H., 1892, "Les Méthodes Nouvelles de la Mécanique Celeste", Gauthier-Vilars, Paris.

Romero Gomez, M., Masdemont, J.J., Athanassoula, E. and Garcia Gomez, C.: 2006, astro-ph/0603124

Schwarz, M.P.: 1984, Mon. Not. R. Astr. Soc. 209, 93.

Schwarz, M.P.: 1985, Mon. Not. R. Astr. Soc. 212, 677.
Sellwood, J.A.: 2000, Astrophysics and Space Science 272, 31.

Smale, S: 1967, Bull. Am. Math. Soc. 73, 747.

Sparke, L.S. and Sellwood, J.A.: 1987, Mon. Not. R. Astr. Soc. 225, 653.

Voglis, N., Contopoulos, G., and Efthymiopoulos, C, 1998, Phys. Rev. E, 57, 372.

Voglis, N., Contopoulos, G., and Efthymiopoulos, C, 1999, Cel. Mech. Dyn. Astr., 73, 211.

Voglis, N., Kalapotharakos, C., and Stavropoulos, G., 2002, Mon. Not. R. Astr. Soc., 337, 619.

Voglis. N.: 2003, Mon. Not. R. Astr. Soc. 344, 575.

Voglis, N., Stavropoulos, G., and Kalapotharakos, C., 2006, MNRAS, (in press). 


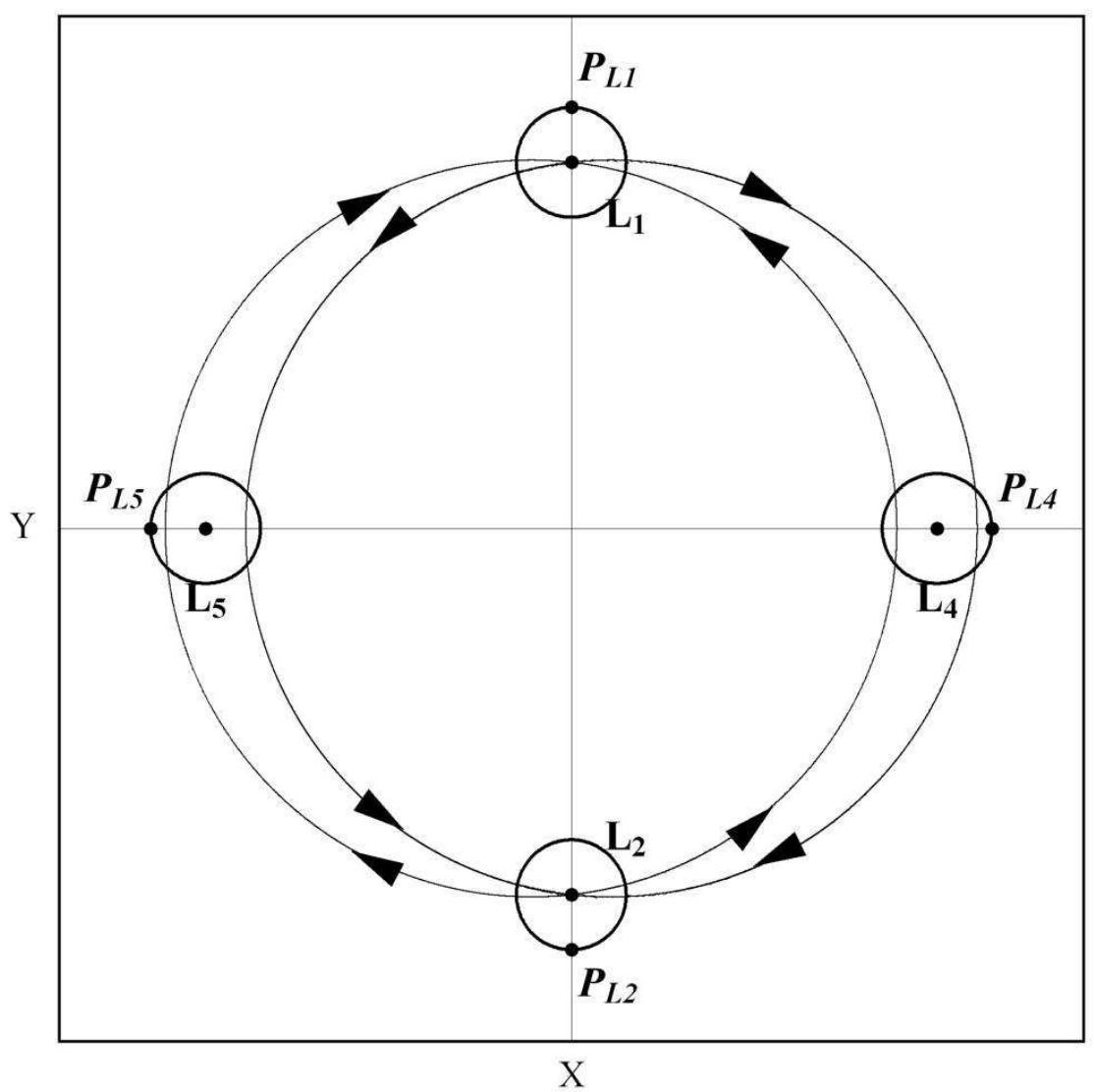

Figure 1. Short period orbits around the Langrangian equilibria $L_{1,2}$ (unstable) and $L_{4,5}$ (stable). The curves with arrow correspond to the equipotential passing from $L_{1,2}$. The arrows indicate the sense of rotation inside and outside corotation. 
(a)

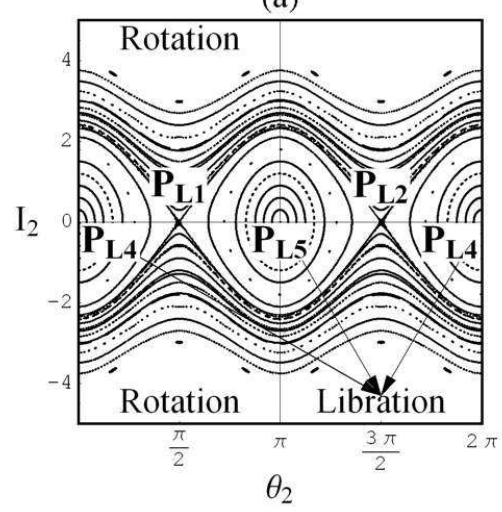

(c)

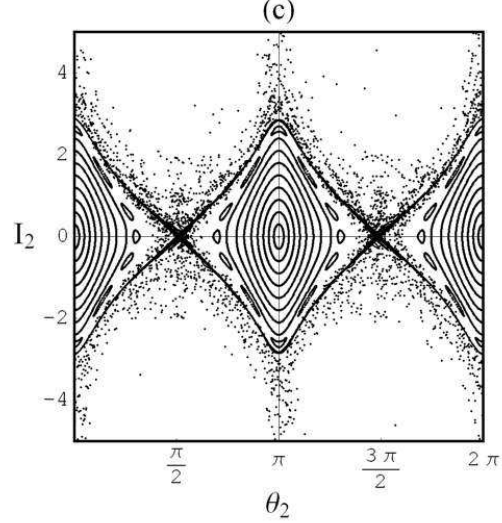

$\mathrm{I}_{2}$
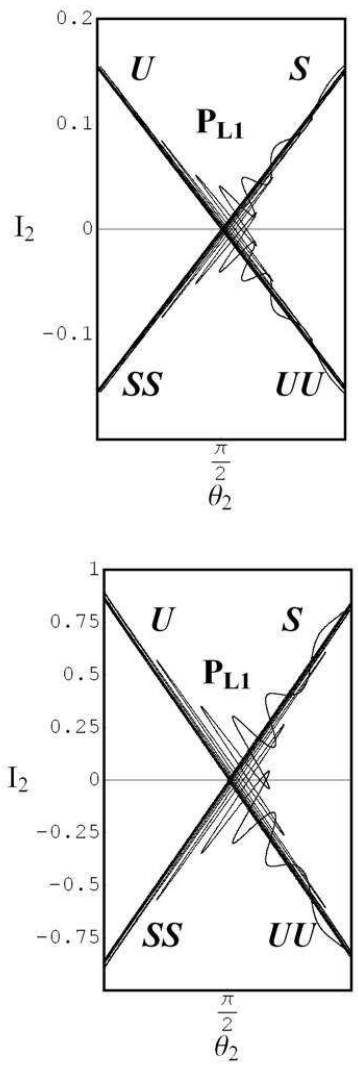

(b)

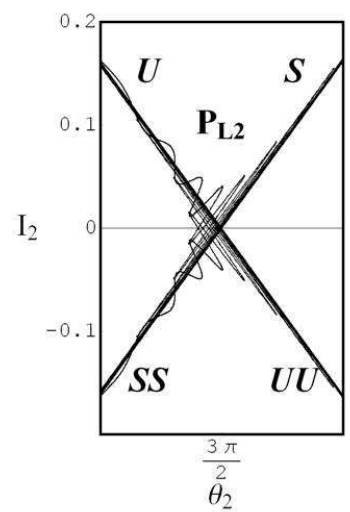

(d)

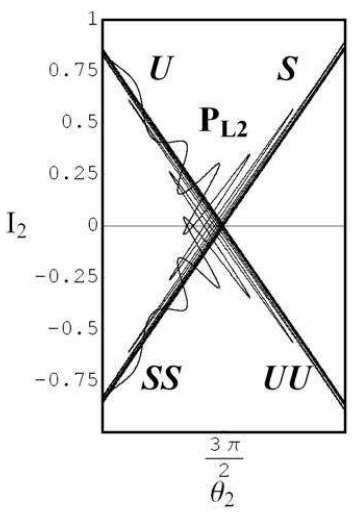

Figure 2. The Poincaré surface of section $\left(I_{2}, \theta_{2}\right)$ for $\theta_{1}=2 k \pi, k=0,1,2, \ldots$ in the Hamiltonian (3) with $\kappa_{*}=3.23414 \times 10^{-1}$, $\alpha_{*}=-2.90577 \times 10^{-2}, b_{*}=0, c_{*}=-7.5 \times 10^{-3}, A_{*}=1.897 \times 10^{-2}$ and (a) $\epsilon=10^{-3},(\mathrm{c}) \epsilon=10^{-2}$. In both cases the value of the Jacobi constant is set equal to $E_{J}=-1.897 \times 10^{-2}$. The stable and unstable manifolds of the periodic orbits $P_{L 1,2}$ (unstable fixed points in (a),(c)) are shown in (b) and (d) respectively.
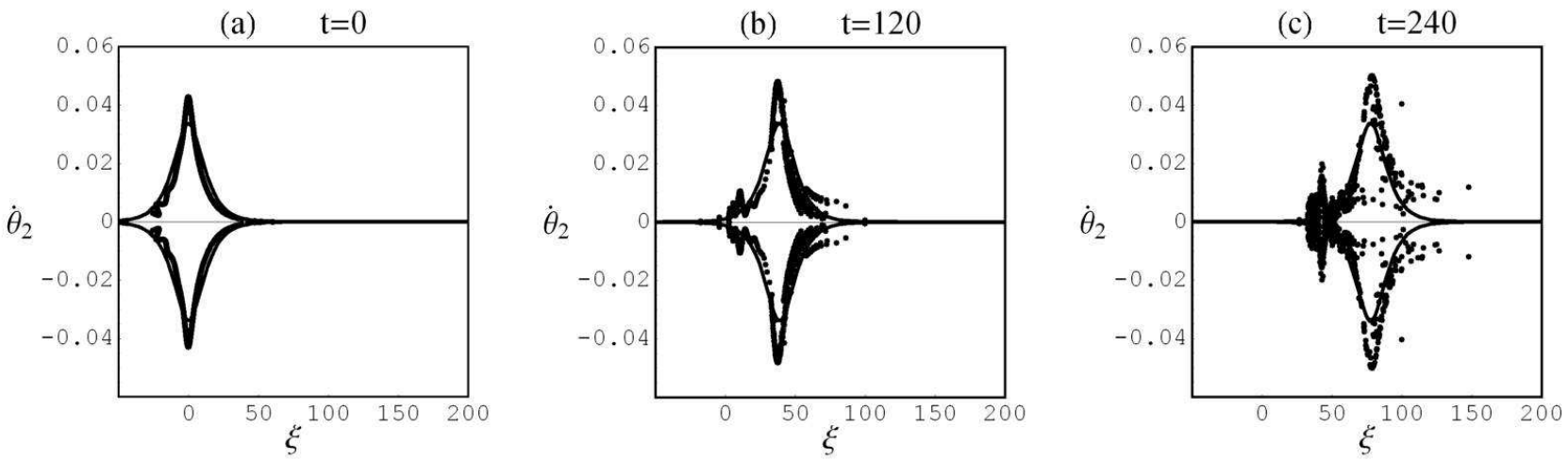

Figure 3. Comparison of the theoretical soliton-type solution given by Eq.(13),(solid line) with the corresponding numerical relation for 200 points along the unstable manifold U emanating from $P_{L 1}$ in the Hamiltonian (3) with parameters as in Fig. 2d. The different panels correspond to different time snapshots, namely a) $t=0$, b) $t=120$, c) $t=240$. 
(a)

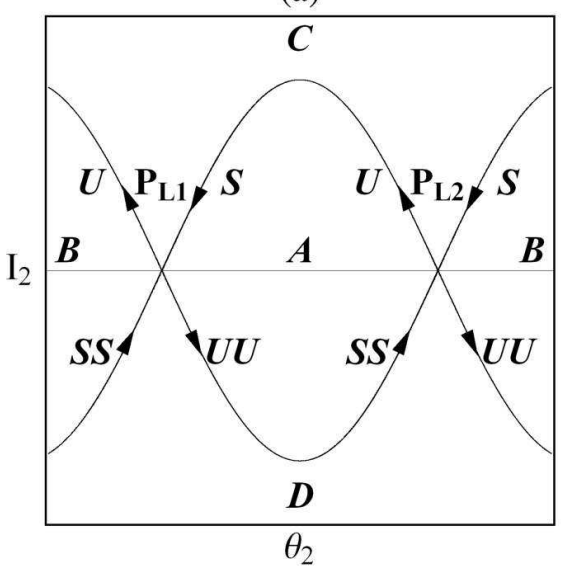

(c)

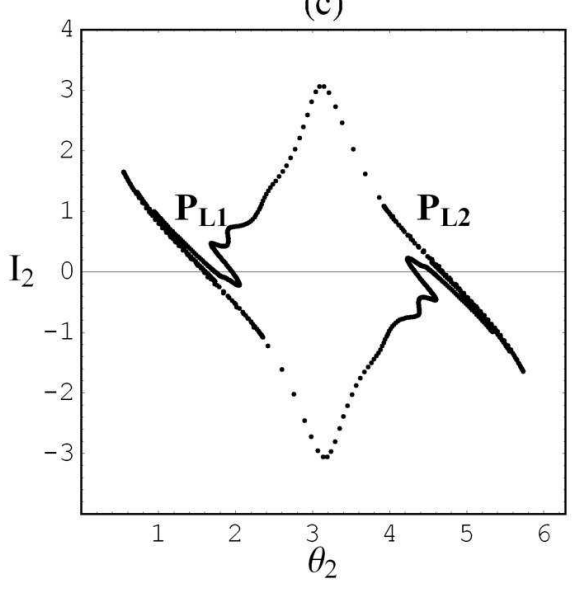

(e)

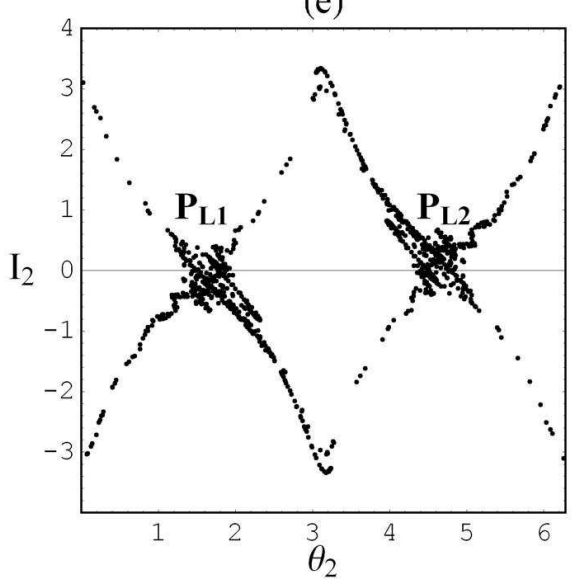

(b)

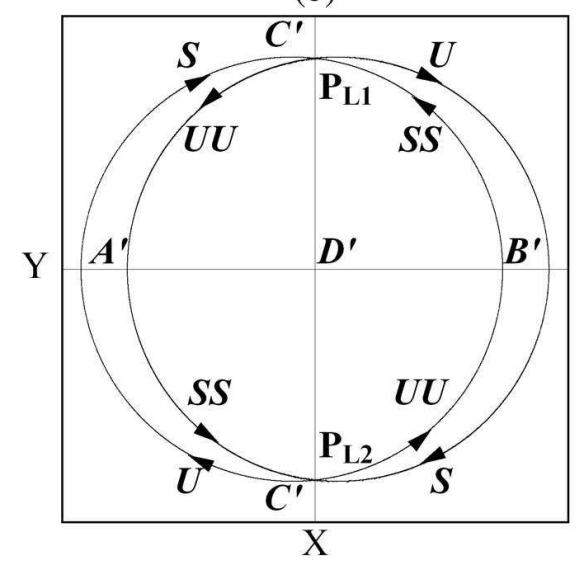

(d)

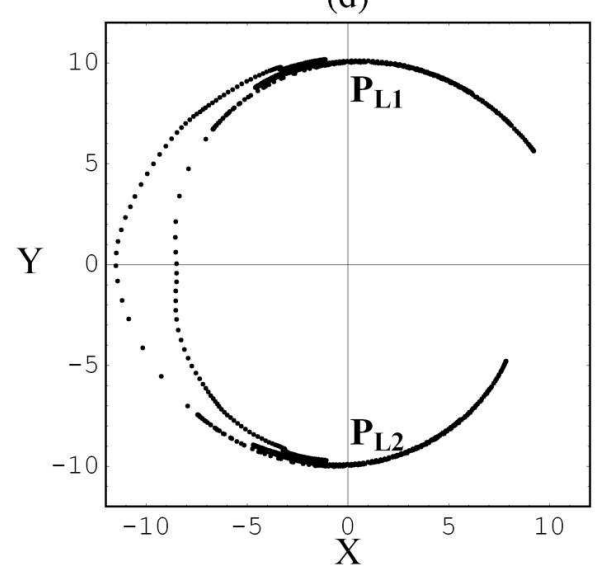

(f)

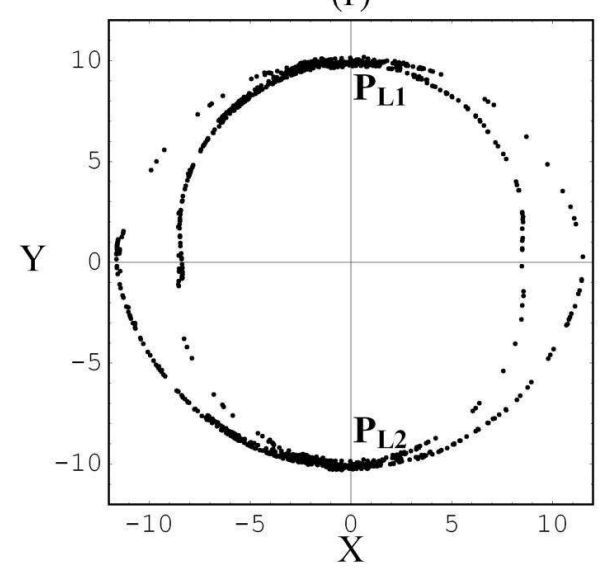

Figure 4. Schematic representation of the isomorphy between a) the phase portrait in the Poincaré section $\left(I_{2}, \theta_{2}\right)$, and b) configuration space. The regions A,B,C,D in (a) correspond to $\mathrm{A}^{\prime}, \mathrm{B}^{\prime}, \mathrm{C}^{\prime}, \mathrm{D}^{\prime}$ in (b). The arrows indicate the directions of the stable and unstable manifolds emanating from $P_{L 1,2}$ in phase space and configuration space. The manifolds emanating from $P_{L 1,2}$ are shown in phase space $(\mathrm{c}, \mathrm{e})$, and configuration space $(\mathrm{d}, \mathrm{f})$ for a short time (four iterations of an initial segment $\left.d s=10^{-4}\right),(\mathrm{c}, \mathrm{d})$ and longer time $(12$ iterations), (e,f). 
(a)

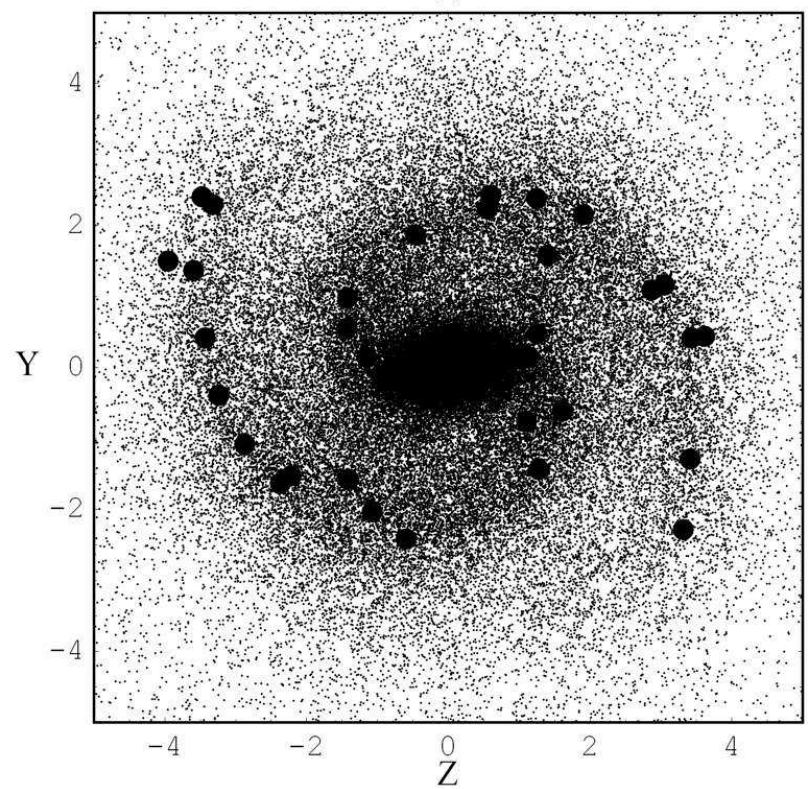

(b)

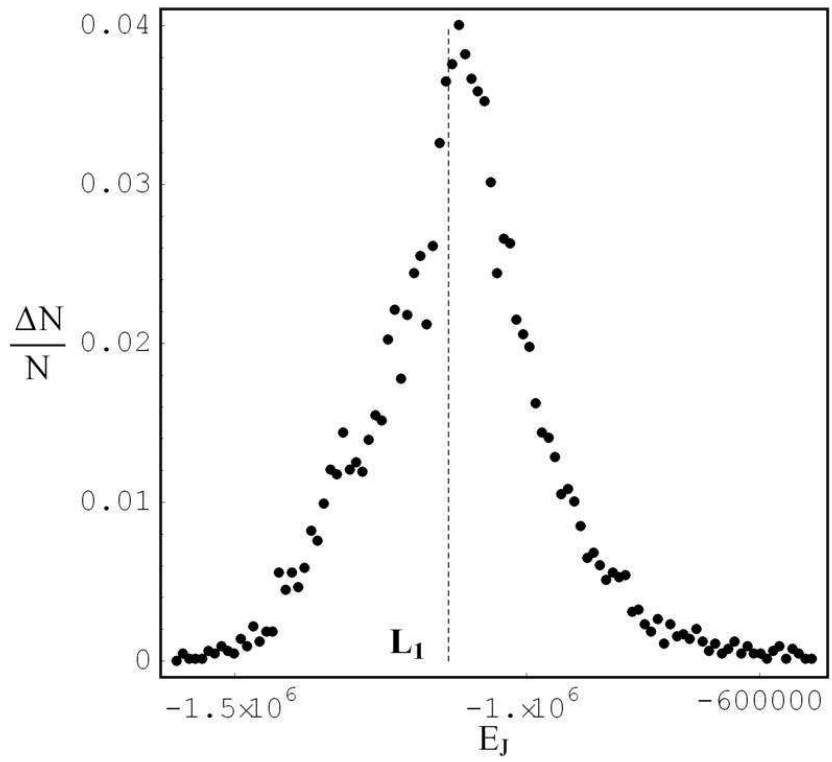

Figure 5. a) Projection of the particles of the N-Body simulation described in section 3 on the $(y, z)$ plane, at the time snapshot $t=47$. In this projection the galaxy rotates counterclockwise. The thick dots are the maxima of the surface density calculated with a division of the system in concentric rings (see text for details). b) The distribution of the Jacobi constant values (in units of the N-body code) for the particles located in small bins $\Delta r \Delta \theta$ centered at the thick dots of (a). The dashed vertical line indicates the Jacobi constant value for $L_{1}$.

(a)

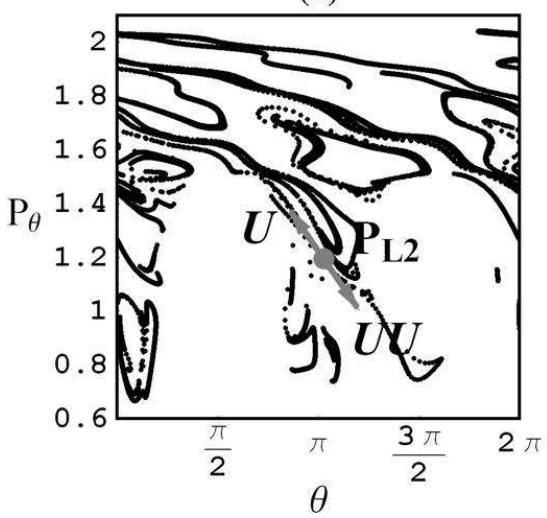

(b)

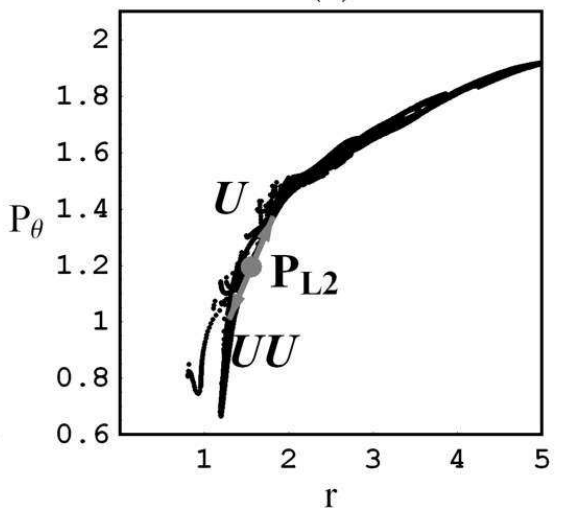

(c)

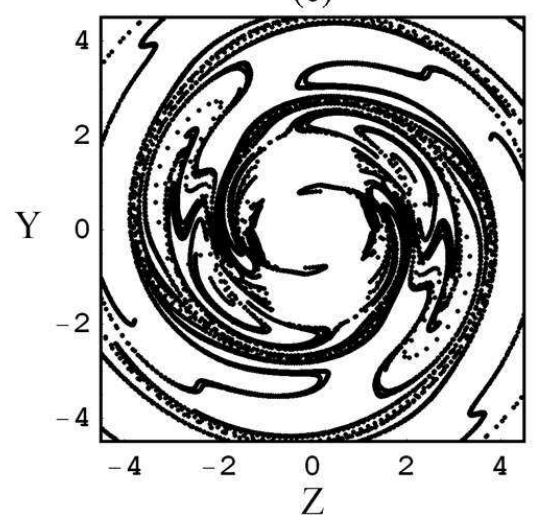

Figure 6. Projections of the apocentric manifolds: (a) on the surface of section plane $\left(\theta, p_{\theta}\right),(\mathrm{b})$ on the plane $\left(r, p_{\theta}\right)$ and $(\mathrm{c})$ on the configuration space $(r, \theta)$ or $(z, y)$. In (a) and (b) the unstable fixed point $P_{L 2}$ and the corresponding initial directions of the manifolds $\mathrm{U}$ and UU are also shown. In (c) the projection of the symmetric manifolds, emanating from the fixed point $P_{L 1}$, is shown together with the manifolds from $P_{L 1}$. The monotonic (on average) dependence of $r$ on $p_{\theta}$ shown in (b) produces a near-isomorphy between (a) and (c). 
(a)

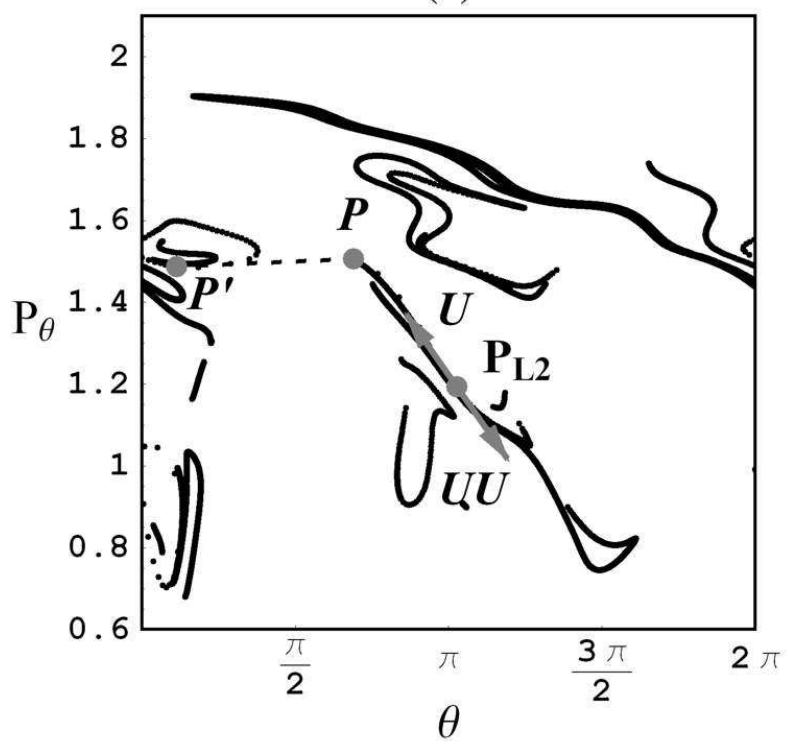

(c)

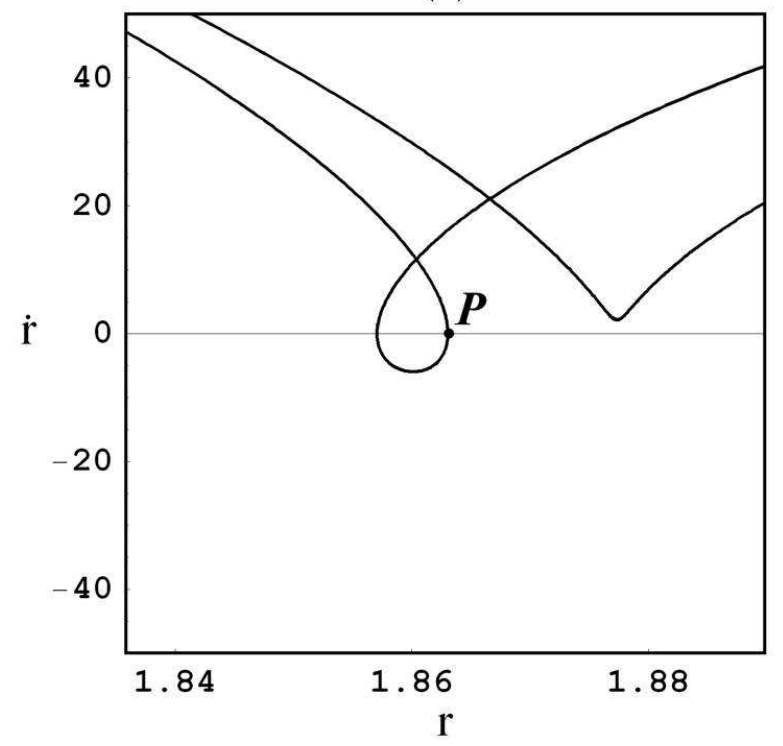

(b)

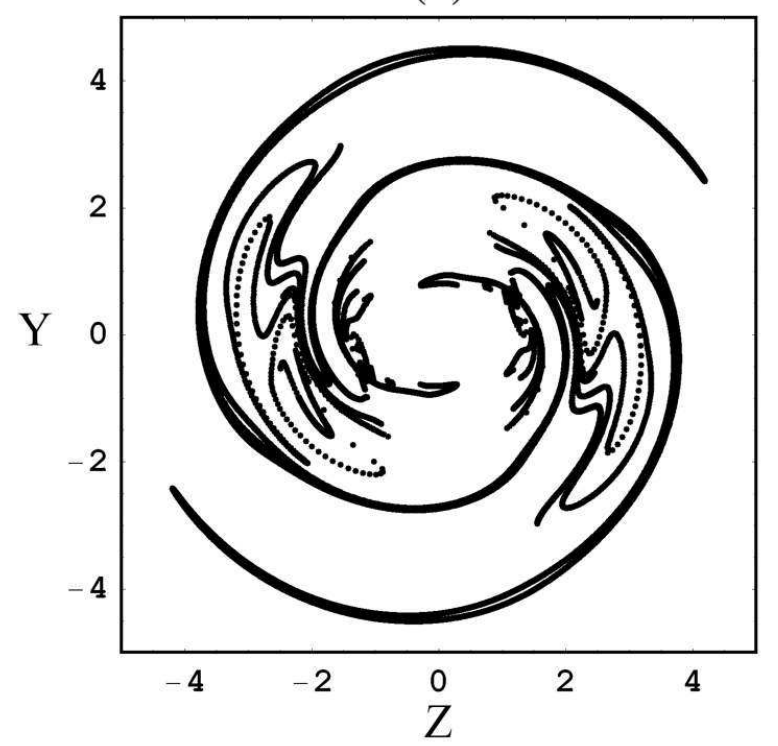

(d)

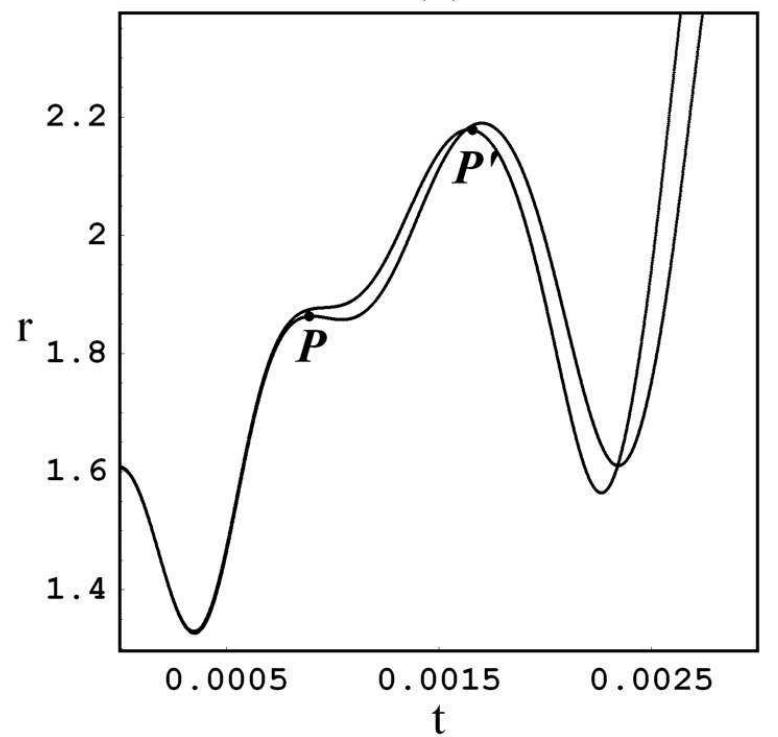

Figure 7. a) Unstable invariant manifolds of the unstable periodic orbit $P_{L 2}$ in the Poincaré section $\left(\theta, p_{\theta}\right), \dot{r}=0, \ddot{r}<0$ of the potential of the N-Body simulation, at the time snapshot $t=47$. The initial directions are marked U and UU. The initial segments along $\mathrm{U}$ and $\mathrm{UU}$, of length $d s=10^{-4}$, are populated with 10000 points each. The figure shows the image of these points after six iterations of the Poincaré map. The Jacobi constant is $E_{J}=-1.125 \times 10^{6}$. b) The manifolds of (a) projected in configuration space, together with the symmetric manifolds with respect to the center. c) The evolution $\dot{r}$ vs. $r$ for two orbits which give the points of intersection $P$ and $P^{\prime}$ in the Poincaré section. d) The time evolution of $r(t)$ for the same pair of orbits. 
(a)

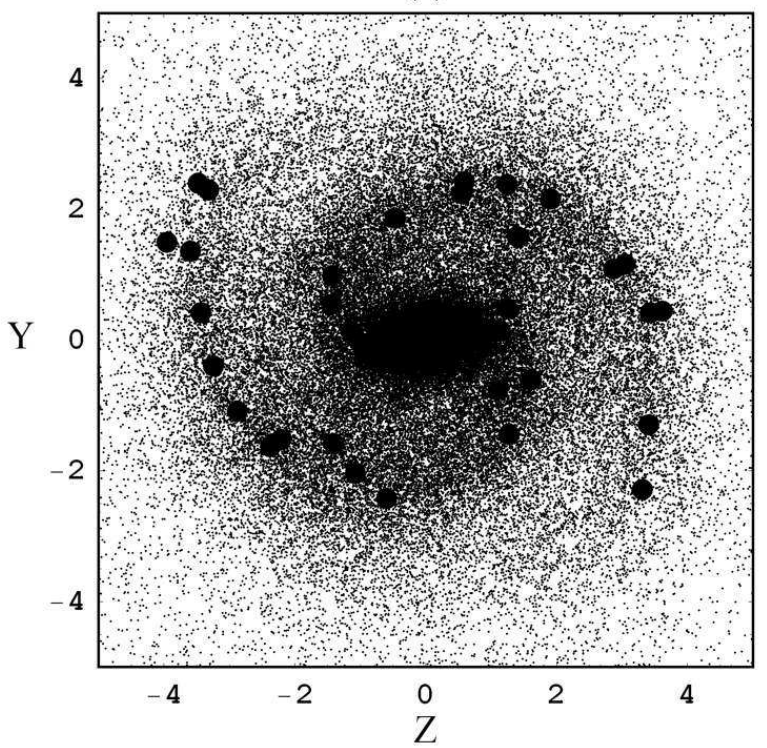

(c)

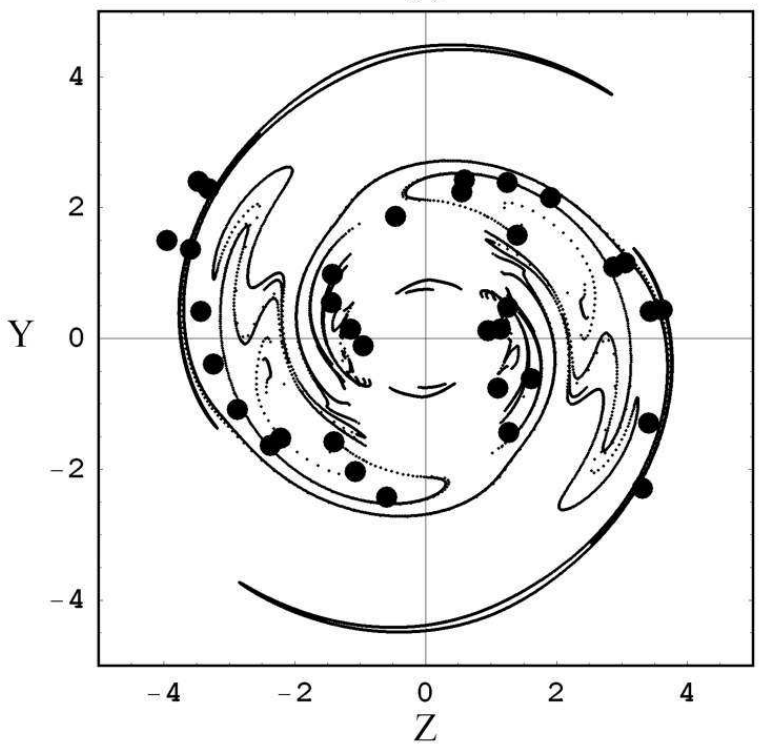

(b)

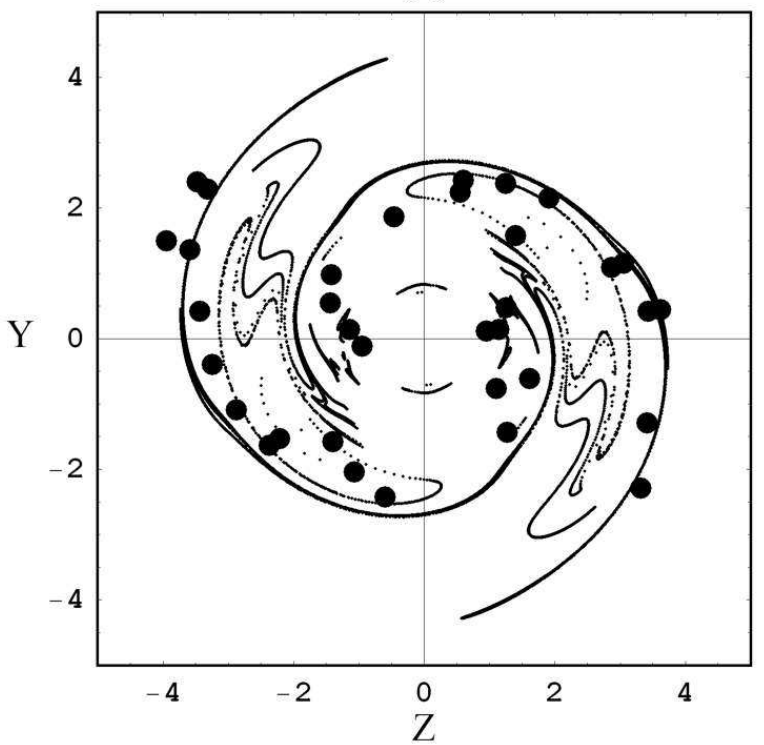

(d)

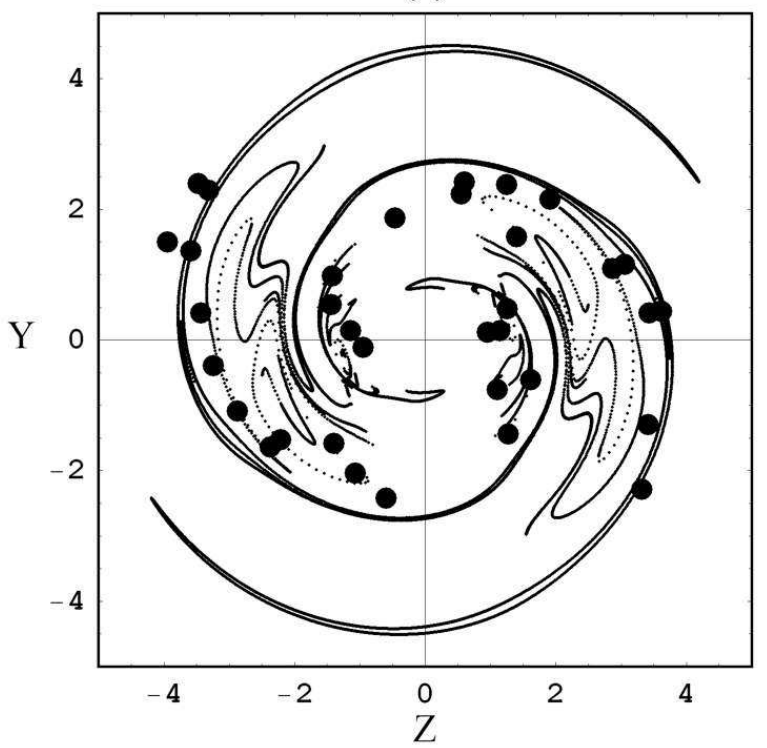

Figure 8. a) Same as in figure 5a. The form of the spiral (thick dots) is compared with the forms of the unstable manifolds emanating from the unstable periodic orbits $P_{L 1,2}$ for three different values of the Jacobi constant, namely b) $\left.E_{J}=-1.131 \times 10^{6}, \mathrm{c}\right) E_{J}=-1.128 \times 10^{6}$, and d) $E_{J}=-1.125 \times 10^{6}$. 
(a)

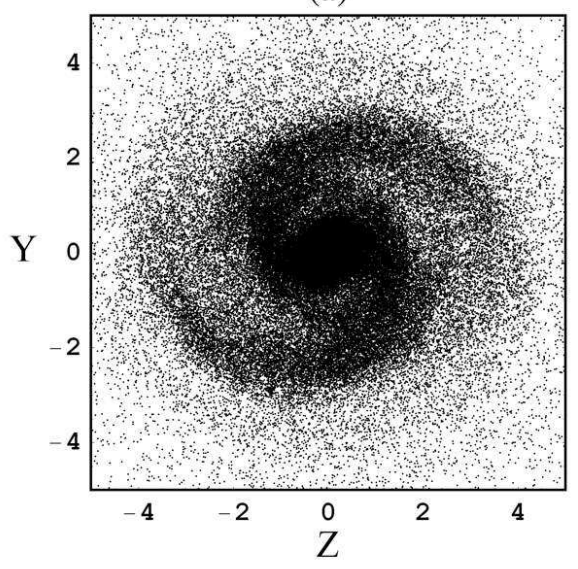

(c)

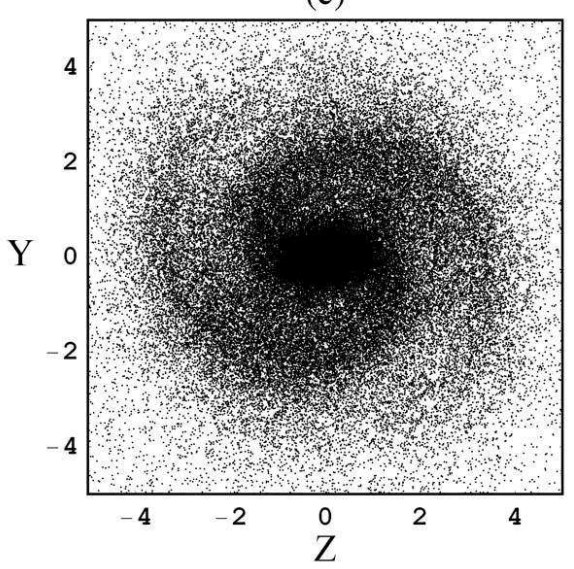

(e)

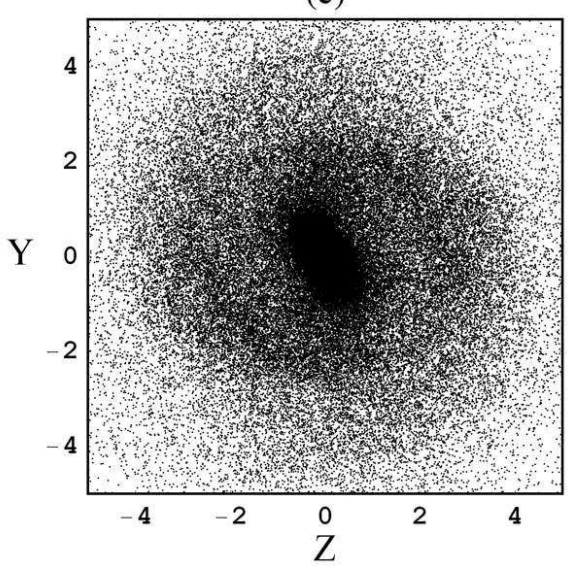

(b)

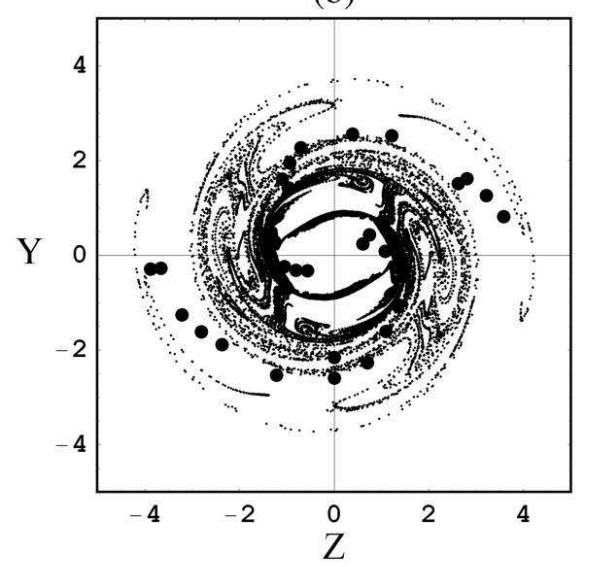

(d)

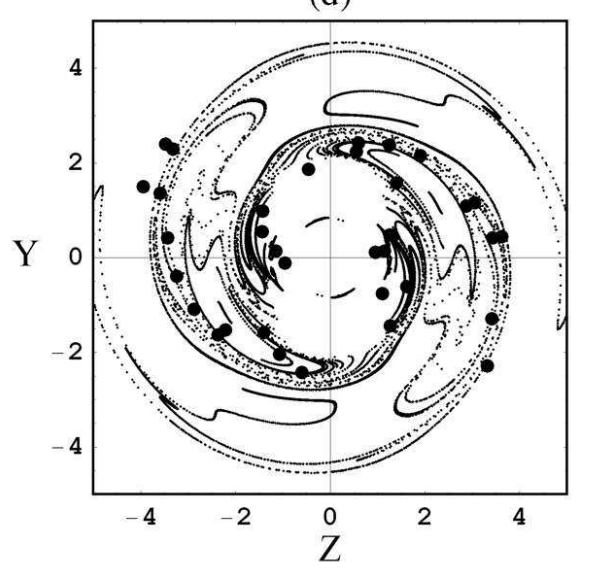

(f)

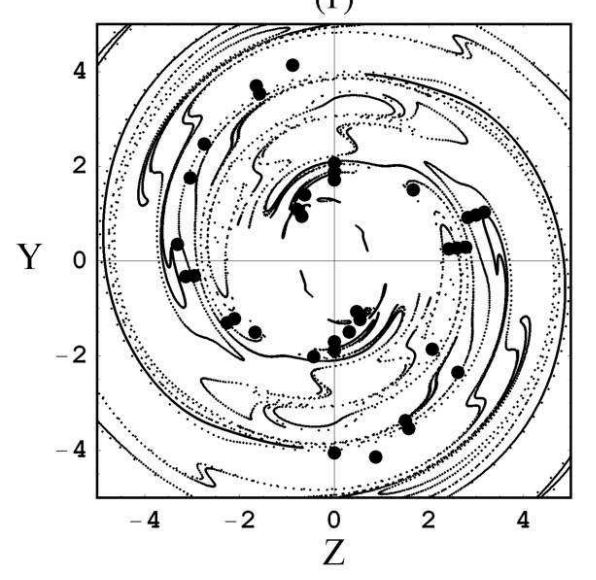

Figure 9. The projection of the N-Body system on the $(y, z)$ plane at three different snapshots: a) $t=18$, c) $t=47$, and e) $t=74$. The comparison of the spiral pattern with the unstable invariant manifolds emanating from $P_{L 1,2}$ is made in (b), (d) and (f) respectively. The corresponding Jacobi constant values at which the manifolds are calculated is $E_{J}=-1.25 \times 10^{6}$ for $(\mathrm{b}), E_{J}=-1.131 \times 10^{6}$ for $(\mathrm{d})$ and $E_{J}=-1.085 \times 10^{6}$ for (f). The 8-th manifolds' iteration is shown. 

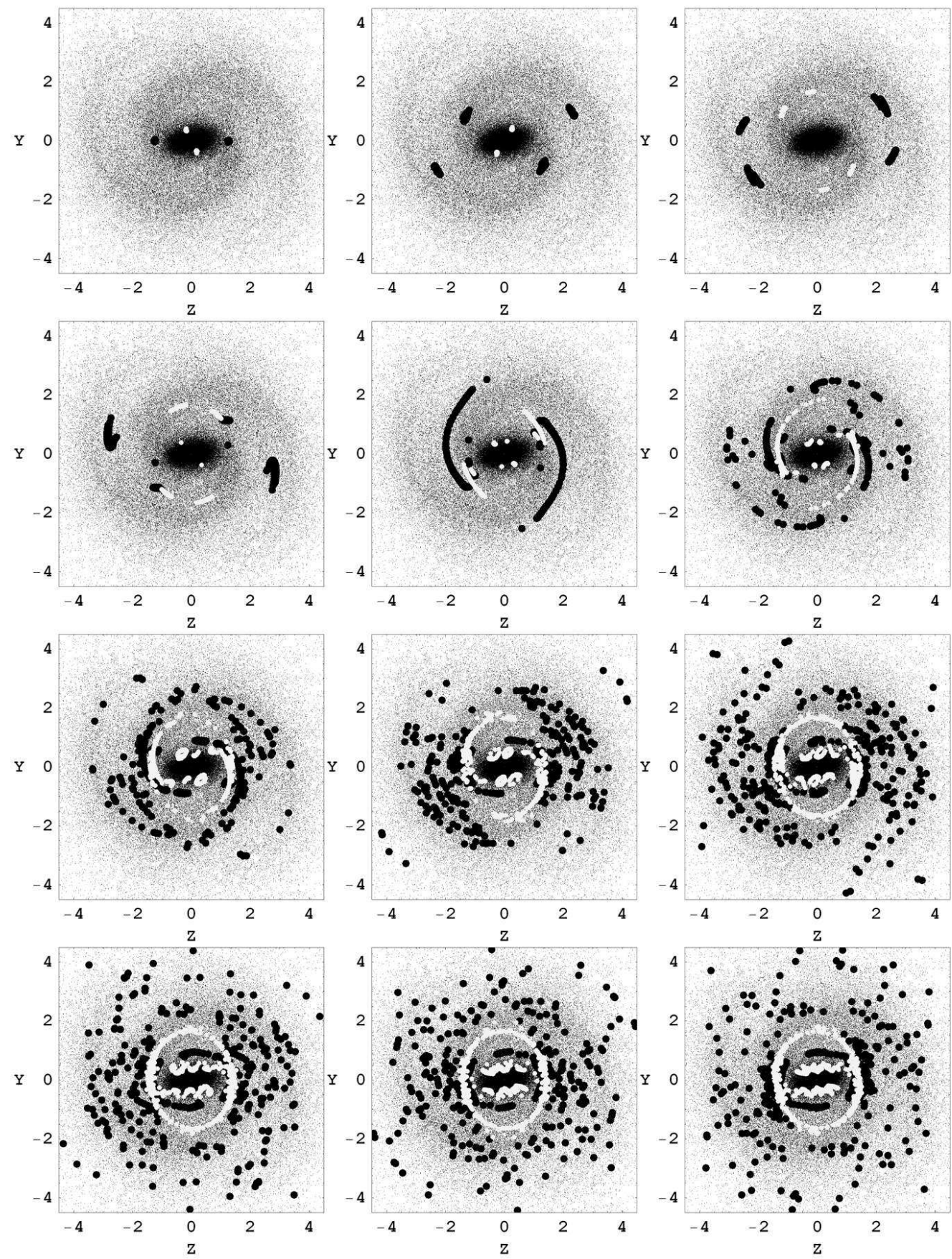

Figure 10. Twelve successive Poincaré consequents of the apocenters (thick black dots) and the pericenters (thick white dots) of 600 orbits with initial conditions on the unstable manifolds $\mathrm{U}$ emanating from the fixed points $P_{L 1,2}$ (300 orbits for each fixed point). The first panel corresponds to the Poincaré consequent at the time when the apocenters start leaving the neighborhood of the fixed points. The phases of the apocenters of these orbits remain well correlated for at least 9 successive passages. The phases of the pericenters are well correlated for much longer. The pericenters tend to form a double-ring surrounding the bar and the stable Lagrangian points $L_{4}$, $L_{5}$ and cooperate with apocenters to support a chaotic shell surrounding the bar, but also an azimuthal arc of the spiral arms near $L_{1}$, $L_{2}$, outside corotation. Spiral arms at larger azimuthal angles and larger radii can be supported only by apocenters. 